\title{
First Results from the THEMIS mission
}

V. Angelopoulos ${ }^{1}$, D. Sibeck ${ }^{2}$, C. W. Carlson ${ }^{3}$, J. P. McFadden ${ }^{3}$, D. Larson ${ }^{3}$, R. P. Lin $^{3}$, J. W. Bonnell ${ }^{3}$, F. S. Mozer ${ }^{3}$, R. Ergun ${ }^{4}$, C. Cully ${ }^{5}$, K. H. Glassmeier ${ }^{6}$, U. Auster ${ }^{6}$, A. Roux ${ }^{7}$, O. LeContel ${ }^{7}$, S. Frey ${ }^{3}$, T. Phan $^{3}$, S. Mende ${ }^{3}$, H. Frey ${ }^{3}$, E. Donovan $^{8}$, C. T. Russell ${ }^{1}$, R. Strangeway ${ }^{1}$, J. Liu ${ }^{1}$, I. Mann ${ }^{9}$, J. Rae ${ }^{9}$, J. Raeder ${ }^{10}$, X. Li ${ }^{4}$, W. Liu ${ }^{4}$, H. J. Singer ${ }^{11}$, V. A. Sergeev ${ }^{12}$, S. Apatenkov ${ }^{12}$, G. Parks ${ }^{3}$, M. Fillingim $^{3}$ and J. Sigwarth ${ }^{2}$.

${ }^{1}$ IGPP/ESS UCLA, Los Angeles, CA 90095-1567, U.S.A

${ }^{2}$ NASA/GSFC, Code 674, Greenbelt, MD 20771, U.S.A

${ }^{3}$ Space Sciences Laboratory, UCB, Berkeley, CA 94720-7450, U.S.A

${ }^{4}$ LASP, University of Colorado, Boulder, CO 80303, U.S.A

${ }^{5}$ Swedish Institute of Space Physics,Upsala, SE 751 21, Sweden

${ }^{6}$ TUBS, Braunschweig, D-38106, Germany

${ }^{7}$ CETP/IPSL, 10-12 Avenue de l' Europe, 78140 Velizy, France

${ }^{8}$ Dept. of Physics and Astronomy, University of Calgary, AB T2N 1N4, Canada

${ }^{9}$ Dept. of Physics, University of Alberta, AB T6G 2J1, Canada

${ }^{10}$ Space Science Center, University of New Hampshire, NH 03824, U.S.A

${ }^{11}$ NOAA/Space Environment Laboratory, Boulder, CO 80303, U.S.A

${ }^{12}$ Institute of Physics, University of St. Petersburg, St Petersburg, 198904, Russia

Abstract. THEMIS was launched on February 17, 2007 to determine the trigger and large-scale evolution of substorms. During the first seven months of the mission the five satellites coasted near their injection orbit to avoid differential precession in anticipation of orbit placement, which started in September 2007 and led to a commencement of the baseline mission in December 2007. During the coast phase the probes were put into a string-of-pearls configuration at $100 \mathrm{~s}$ of $\mathrm{km}$ to $2 \mathrm{R}_{\mathrm{E}}$ along-track separations, which provided a unique view of the magnetosphere and enabled an unprecedented dataset in anticipation of the first tail season. In this paper we describe the first THEMIS substorm observations, captured during instrument commissioning on March 23, 2007. THEMIS measured the rapid expansion of the plasma sheet at a speed that is commensurate with the simultaneous expansion of the auroras on the ground. These are the first unequivocal observations of the rapid westward expansion process in space and on the ground. Aided by the remote sensing technique at energetic particle boundaries and combined with ancillary measurements and MHD simulations, they allow determination and mapping of space currents. These measurements show the power of the THEMIS instrumentation in the tail and the radiation belts. We also present THEMIS Flux Transfer Events (FTE) observations at the magnetopause, which demonstrate the importance of multi-point observations there and the quality of the THEMIS instrumentation in that region of space. 
Keywords: THEMIS, magnetosphere, substorms, radiation belts, magnetopause

PACS codes: 94.30.-d; 94.30.cl; 94.30.cb; 94.30.ch; 94.30.cj; 94.30.C-;

94.30.cp; 94.30.Lr; 94.30.Va; 94.30.Xy; 96.50.Fm. 
First Results from the THEMIS mission 


\section{Introduction}

After a successful launch on February 17, 2007, and instrument commissioning by mid-March 2007, THEMIS’s in-flight compatibility testing took place over a period of several weeks. Probe orbits were assigned constellation positions on March 27, followed by EFI boom deployments on probe C by May 16 and on probes D and E by June 7. During the first seven months of the mission, the five satellites coasted very near their injection orbit to avoid differential precession, in anticipation of a 3 month-long orbit placement maneuver period that would take the probes in their final position to the first baseline tail season. Probe placement maneuvers commenced on September $1^{\text {st }}$ and ended on December $4^{\text {th }}$. Probe B's EFI booms were deployed after the main orbit maneuvers finished on Nov 17, and Probe A's EFI booms are expected to be deployed by January 12, 2008, following the final positioning of the replacement probe, TH-A (P5).

In the period between instrument commissioning and final orbit placement, the THEMIS probes were in a string-of-pearls configuration. In the first three months the string-of-pearls separations came about due to the natural orbit dispersions from the launch vehicle, with TH-C leading and E trailing at distances of $\sim 2 \mathrm{R}_{\mathrm{E}}$ from each other at apogee, while $\mathrm{D}, \mathrm{B}$, and A were at $\sim 1000 \mathrm{~km}$ separations between each other in the middle of the constellation. In the ensuing three months the probe positions were re-organized along their tracks, such that three middle probes were the ones equipped with EFI - deployed instruments (C, D, E) at $~ 100 \mathrm{~km}$ separation, whereas B and A were leading and trailing respectively at separations of $\sim 2 \mathrm{R}_{\mathrm{E}}$ from each other. The orbit configuration during the coast phase is shown in Angelopoulos (2008), and it is further detailed in Sibeck et al. (2008) and in Frey et al. (2008).

Before leaving the tail on March 23, 2007 between 11:10 and 15:10UT, all THEMIS instruments were ON, and data collection was commanded into "Fast Survey” mode, which enables storage and transmission of high time resolution ion distributions and fields waveforms. Two substorms were captured from a unique vantage point at the dusk sector. Simultaneous observations from the POLAR satellite captured the evolution of the first substorm in global imaging, which complemented the ground-based imaging from the THEMIS GBOs. MHD 
simulations of the event, using actual solar wind input, performed mapping that differs from statistical models and provides the best agreement with the data. The mapping enables interpretation of THEMIS in the context of the substorm current wedge as derived from modeling of ground-based magnetometer data. Timing using $\mathrm{Pi} 2 \mathrm{~s}$ and $\mathrm{PiBs}$ is consistent with that derived from imaging, and provides onset and intensification times to within a fraction of a minute. This paper provides the global context of the event and reports on a major finding from the event, namely the first simultaneous and commensurate observations of the expansion of the westward traveling surge in space and on the ground.

The optimal string-of-pearls configuration, at along-track separations of $100 \mathrm{~s}$ of $\mathrm{km}$ between probes on which EFIs were deployed and on the order of $1 \mathrm{R}_{\mathrm{E}}$ between the leading and trailing probes, provided an unprecedented dataset in anticipation of the first tail season. In this paper we also present THEMIS measurements at the magnetopause, which demonstrate the efficacy of multi-point observations from such a configuration in the dayside, and bespeak the quality of the THEMIS instrumentation.

\section{THEMIS first light: March 23, 2007, -11:00UT substorm}

At substorm onset, auroras expand poleward and westward (Akasofu 1976). In space the substorm current wedge forms at the meridian of the substorm activation (Atkinson 1967; McPherron et al. 1973). The westward portion of the auroral expansion is associated with the most intense field-aligned currents, as observed by low-altitude satellites (Hoffman et al. 1994). The field-aligned currents of the current wedge are expected to feed into and out of the expanding aurora. Specifically, the west portion of the substorm current wedge is expected to be responsible for the most intense current above the aurora, while the westward expansion of the two processes should match. These have never been observed to match each other with simultaneous measurements on the ground and in space. The expansion of the substorm current wedge in space has been studied statistically and with fortuitous conjunctions of satellites. Nagai $(1982,1991)$ used GOES satellite magnetometer data from geosynchronous altitudes and showed that the average current wedge expansion speed varies from $<0.5 \mathrm{MLThrs} / \mathrm{min}$ when the satellites are on average $>1 \mathrm{MLT}$ hours away from the substorm meridian, on the order of 1MLThrs/min if the satellites are located close to the 
substorm meridian. These expansion speeds were not well constrained near the substorm meridian due to the longitudinal separation of the GOES satellites (2MLT hrs). They also indicate infinite speed at the substorm meridian due to the reversal in the expansion direction from duskward to eastward at the substorm meridian. The azimuthal expansion of the current wedge has also been studied using fortuitous conjunctions between near-Earth satellites (e.g. Lopez and Lui 1990) and found to take place in abrupt steps, comprised of localized dipolarization events of $1-2 \mathrm{R}_{\mathrm{E}}$ scale size.

The heated plasma within the dipolarized plasma sheet originates in a small (Ohtani et al. 1991) equatorial area $\left(\sim 1 \mathrm{R}_{\mathrm{E}}{ }^{2}\right)$ and has been observed to expand radially both outward (Jacquey et al. 1991; Ohtani et al. 1992) and inward (Ohtani 1998) at $200 \mathrm{~km} / \mathrm{s}$. It has also been observed, on occasion, accompanied by near-Earth fast Earthward plasma flows (Fairfield et al. 1998; Angelopoulos et al. 1999). The relevance of these flows in the buildup of the magnetic flux, which comprises the substorm current wedge, differs in two prominent models of substorms (Lui 1996; Baker et al. 1996). However, the presence of such localized, transport-efficient fast flows in the near-Earth tail, especially further downtail of $10 \mathrm{R}_{\mathrm{E}}$ is well documented (Angelopoulos et al. 1994; Sergeev et al. 1996, Angelopoulos et al. 1997). Those fast Earthward plasma flows are most often quite localized (e.g. Nakamura et al. 2004) and therefore the question arises how such localized flows participate in replenishing the magnetic flux eroded from the day side during the course of the substorm, and how they partake in the azimuthal expansion of the near-Earth dipolarization which extends over many hours of local time.

One attempt to explain the localized $\left(1-2 \mathrm{R}_{\mathrm{E}}\right)$ nature of the fast flows and the extended nature of the substorm current wedge was made in Angelopoulos et al. (1996), in which the incoming flux transport is gradually diverted around the dipolar region, and further fast flows are layered on the outer edges of the substorm current wedge. In that picture, a westward expansion of the substorm current wedge would be accompanied by Earthward and duskward flow, piling additional magnetic flux further to the west. While consistent with single satellite observations available prior to THEMIS, this picture has not been verified by multi-satellite observations. THEMIS's string-of-pearls configuration available from the coast phase of the mission enables such observations for the first time. 
In this paper, we will demonstrate that the expanding westward traveling surge maps in space to a westward propagating dipolarization, which is accompanied by fast Earthward flows near its westward edge.

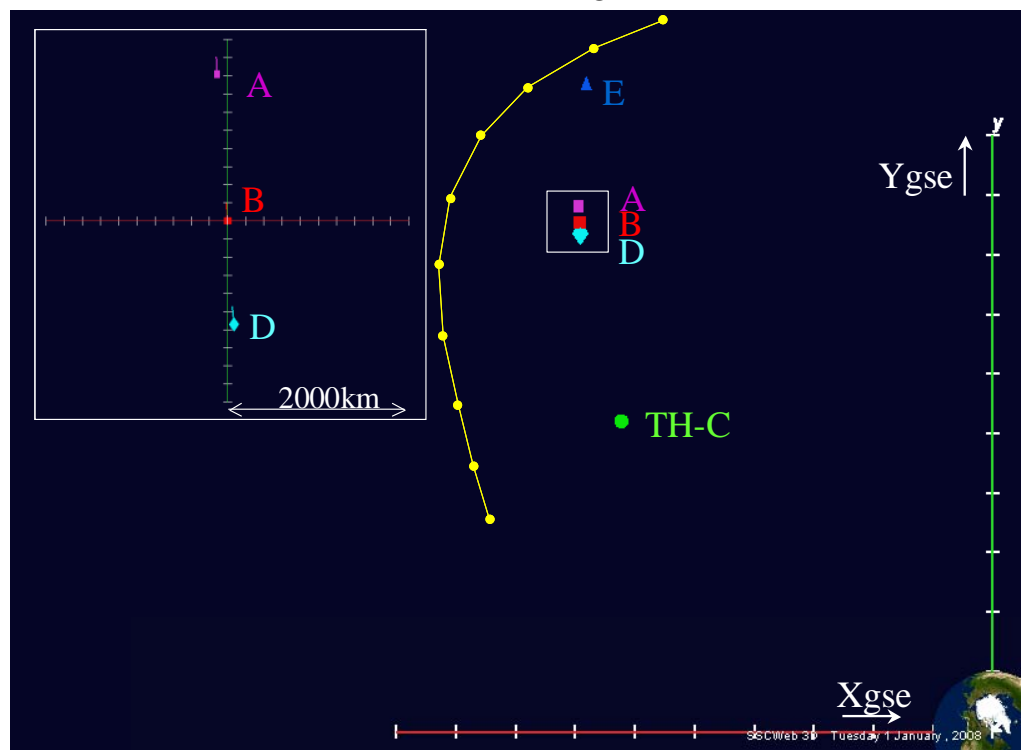

\begin{tabular}{|l|l|l|r|r|}
\hline \multicolumn{1}{|c|}{ Satellite } & \multicolumn{1}{c|}{ Color } & \multicolumn{1}{c|}{ X } & \multicolumn{1}{c|}{$Y$} & \multicolumn{1}{c|}{$Z$} \\
\hline THEMIS-A (P5) & & -6.947 & 8.808 & -2.66 \\
\hline THEMIS-B (P1) & & -6.928 & 8.544 & -2.607 \\
\hline THEMIS-C (P2) & & -6.227 & 5.202 & -1.874 \\
\hline THEMIS-D (P3) & & -6.918 & 8.357 & -2.571 \\
\hline THEMIS-E (P4) & & -6.817 & 10.89 & -3.034 \\
\hline
\end{tabular}

Figure 1. THEMIS probe locations [GSE, $R_{E}$ ] on Mar. 23, 2007, 11:20UT. Insert is an expanded view of the inner probes $A, B, D$, at a different scale, shown by the arrow within. (This product is available at http://sscweb.gsfc.nasa.gov/tipsod ). Note the typical color scheme for THEMIS probes is as shown in the insert. The yellow curve is an equatorial projection of an ionospheric trace on a constant (66deg) magnetic longitude line, at 5deg magnetic longitude increments from 285deg to 330deg using the Tsyganenko (1989) model.

On March 23, 2007, THEMIS was in an ideal position to observe the westward propagation of a substorm (Figure 1). The probes were on the inbound leg of their orbit, in the pre-midnight sector. The upstream solar wind conditions measured by Cluster, as well as by WIND and ACE when time-shifted to the magnetopause location, show that the interplanetary field was southward for an hour prior to 11:08 UT ( $\left.B_{z} \sim-5 n T\right)$. The IMF turned momentarily northward at around 11:08UT at which time the solar wind density, previously around $~ 10$ /cc, abruptly increased by $80 \%$, though the solar wind speed remained steady at $\sim 350 \mathrm{~km} / \mathrm{s}$. An overview of the conditions in the nightside magnetosphere is shown in Figure 2. That figure shows data from TH-B in the standard "overview" plot of the THEMIS data distribution. (Such plots are available routinely on the 
web at $6 \mathrm{hr}$ and 24hr intervals at http://themis.ssl.berkeley.edu, and are automatically produced for arbitrary times using the machine-independent Graphical User Interface of the THEMIS software distribution.)

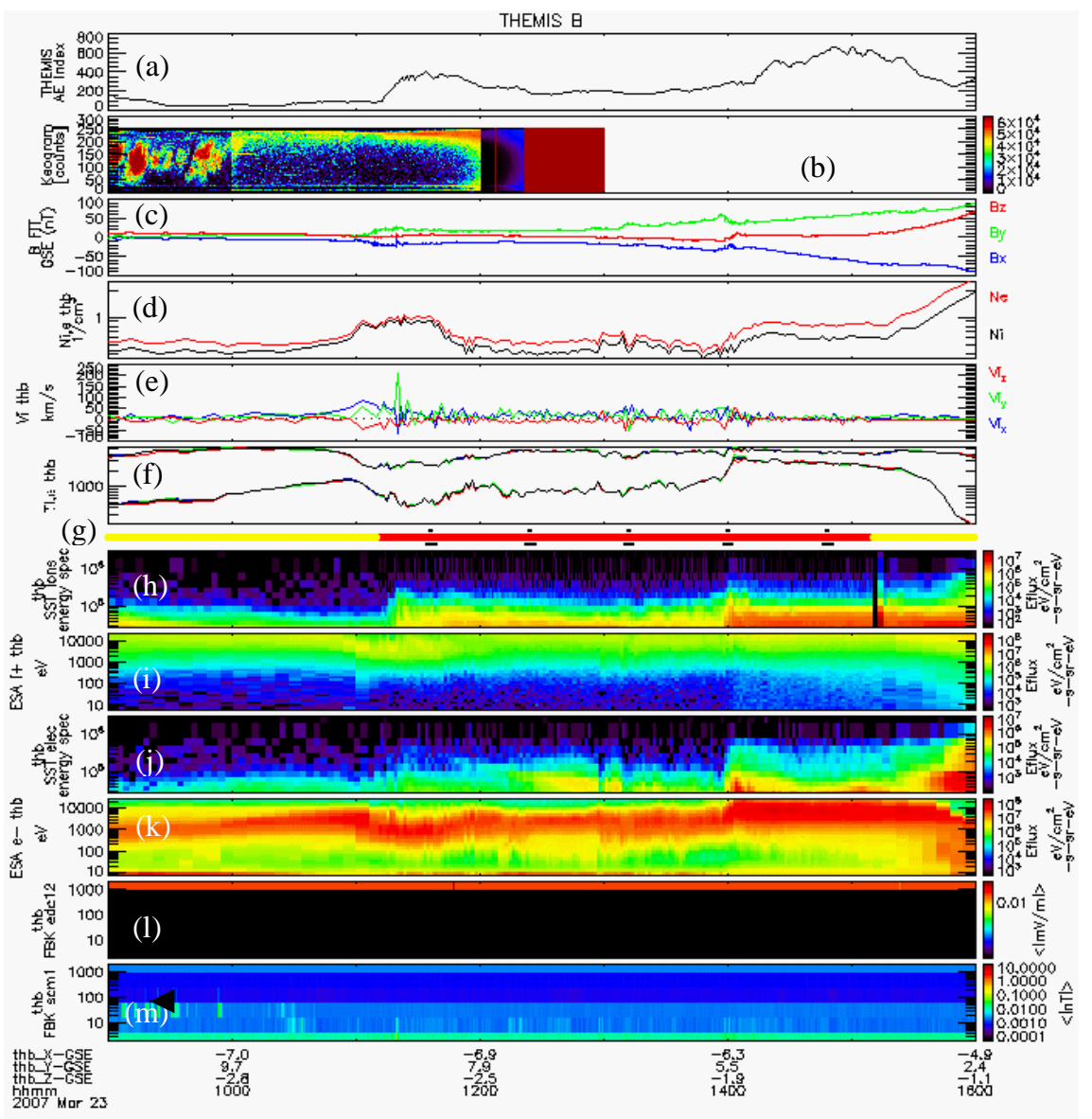

Figure 2. Standard overview plot from THEMIS probe B, on Mar. 23, 2007, 09-16 UT. (a) THEMIS pseudo-AE index, generated from the THEMIS GBOs [Mende et al. 2008], indicating a substorm onset around 11:10UT and another onset at around 14:10 UT; (b) Keogram (north-south stripe of an All Sky Imager camera) from Athabasca GBO [Harris et al. 2008]; (c) Magnetic field [Auster et al. 2008] in GSE coordinates, measured on TH-B; (d) Ion and electron partial densities from the ESA instrument [Carlson et al. 2008; McFadden et al. 2008] which measures the thermal ions and electrons. Note that the electron density computation is affected by the presence of photoelectrons (artificial); this can be removed by special processing, or routinely after the EFI measurements of the spacecraft potential became available; (e) Ion partial flow velocity from the ESA instrument; (f) Ion and electron partial temperatures, from the ESA instrument (shown are the three components of the temperature matrix trace, with the lower trace being the electron temperature); (g) Bar indicating the data collection mode. Yellow: Slow Survey, Red: Fast Survey, Black line underneath: Particle Burst, Black line above: Wave Burst; (h) Ion omni-directional spectrum from the SST instrument (Larson et al. 2008); (i) Ion omni-directional spectrum from the ESA instrument; (j) \& ( $k$ ) electron spectra from the SST and ESA instruments; (j) on-board 
computed wave power spectrum at discrete filter-banks from the EFI instrument (Bonnell et al. 2008; Cully et al. 2008) - with the EFI booms stowed this quantity shows no real data on that day; (k) on-board computed wave power spectrum from the SCM instrument (Roux et al. 2008). Satellite position in GSE coordinates is shown at the bottom.

As is evidenced in the THEMIS pseudo-AE index shown in Figure 2, the THEMIS GBOs recorded two main substorms around 11:10 and 14:10UT. Probe TH-B observed an intensification of the energetic particle fluxes in both instances. THEMIS probes were commanded into time-based burst collection during this early period of the mission (i.e., not using on-board triggers); they were thus bursting simultaneously but not necessarily related to local activity. However, all probes were in fast survey mode, during which spin resolution particle data in high angular resolution were available. This allowed computation of accurate high-time resolution moments of the ion distributions.

During this period, the POLAR satellite collected images of the southern auroral oval and observed the entire sequence of the first substorm. Figure 3 shows a compendium of images for this event from the UVI (Torr et al. 1995) and the VIS (Frank et al. 1995) imagers on-board that satellite. Activations occurred at 10:54UT, 11:10UT and 11:18UT, occurring at 21:00MLT, 01:00MLT and 23:00 MLT respectively. It is evident from the MLT-UT spectrogram of Figure 3 that during the 11:10UT onset the pre-cursor activation site, at 21:00MLT, also intensified. Major substorm intensification expanded poleward very rapidly. By taking the difference between consecutive images from the POLAR UVI instrument, specifically at 11:18:26 UT and 11:19:03 UT (see Figure 3), we determine the longitudinal expansion speed to be approximately 1MLT/min. By 19:40UT the intensification had expanded to $21: 30 \mathrm{MLT}$, and did not progress as much or as fast in the ensuing 3.5 minutes as evidenced by the last image in the series, taken at 11:23:21UT. Although UVI's field of view did not extend much to the west of the 21:00 MLT meridian, the VIS global images at 11:18:45 and 11:23:21 UT do confirm the presence of a continuous auroral oval in the early pre-midnight sector (in fact, continuing all the way through dusk to the day side) and are in agreement with UVI regarding the longitude of the westernmost expansion of the substorm aurora. Since western Alaska midnight is at approximately 11:30UT, the primary 11:10UT onset took place 1 hour to the East 
of Alaska, within the field of view of station Inuvik, and the major intensification over the west coast of Alaska.
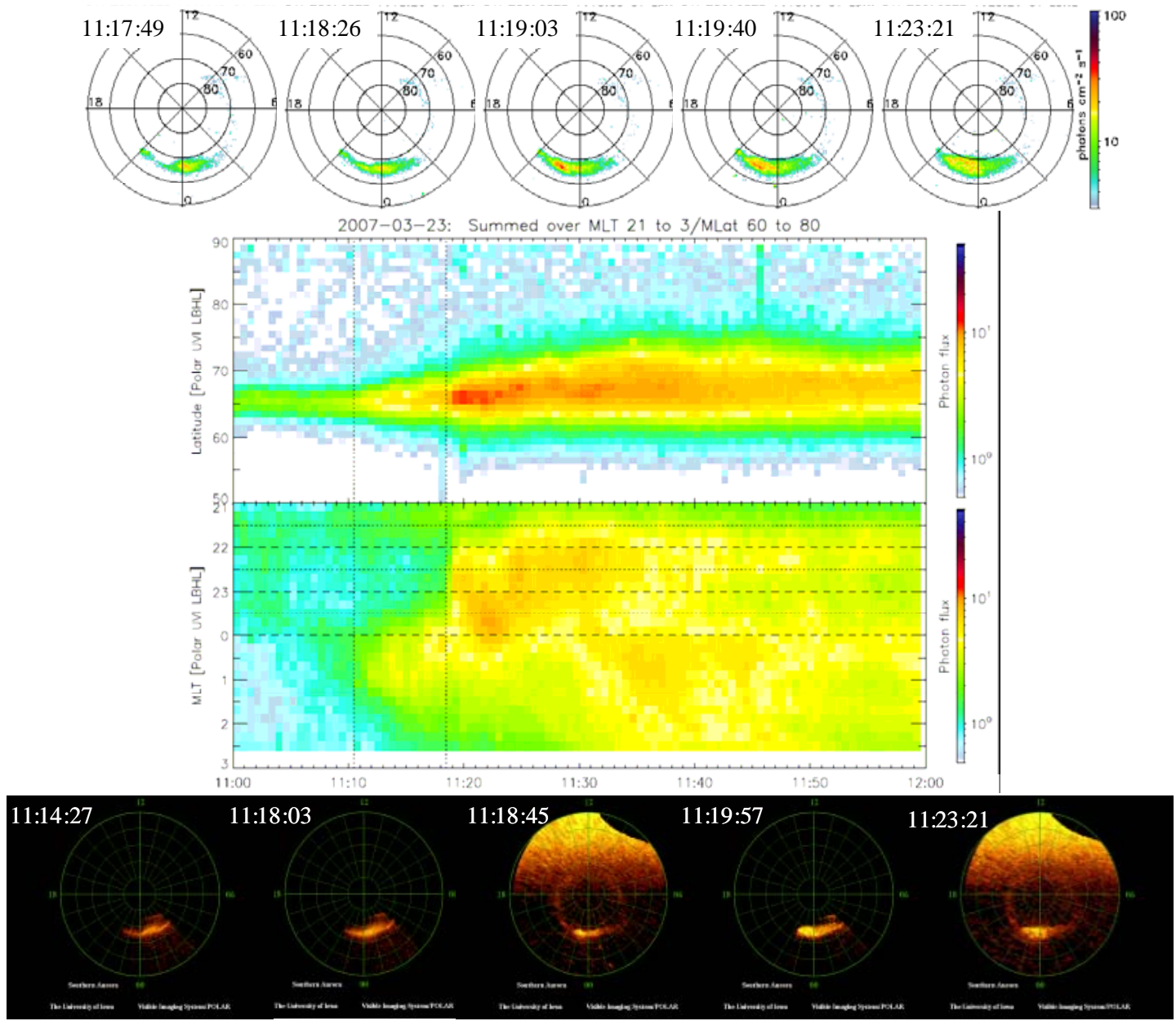

Figure 3. POLAR UVI images (top row) and VIS images (bottom row) from select times during the course of the first substorm on March 23, 2007, projected onto a geomagnetic longitude/latitude map of the Northern Hemisphere. The middle panel, top row shows a latitude-UT spectrogram of the intensity integrated over the pre-midnight sector, while the middle panel bottom row shows an MLT-UT spectrogram of the intensity integrated over auroral latitudes. The times shown next to the images correspond to the beginning times of the image collection. It is evident from the middle panels that a small auroral activation at 21:00 MLT at 10:54UT preceded the major substorm activity. It was followed by a main onset at 01:00 MLT at 11:10 UT, and a major intensification at 23:00 $M L T$, at 11:18:45 UT.

On the ground, the THEMIS GBOs over Alaska and western Canada are able to provide a partial view of the auroral intensification. Figure 4 shows a mosaic from the THEMIS All Sky Imager data in Alaska and western Canada. 
The main onset over Inuvik is evident in that imager, but the activity did not expand from it over Alaska. Smoke from a nearby factory at the Kiana site (since corrected), obscures the field of view at times over western Alaska, but does not prevent a clear identification of a newly formed arc which moved from over the ocean on the west to over western Alaska at 11:18:42 UT. Based on POLAR images, the onset occurred very near the west coast of Alaska and thus its timing was captured quite accurately (within seconds) by the Kiana GBO.

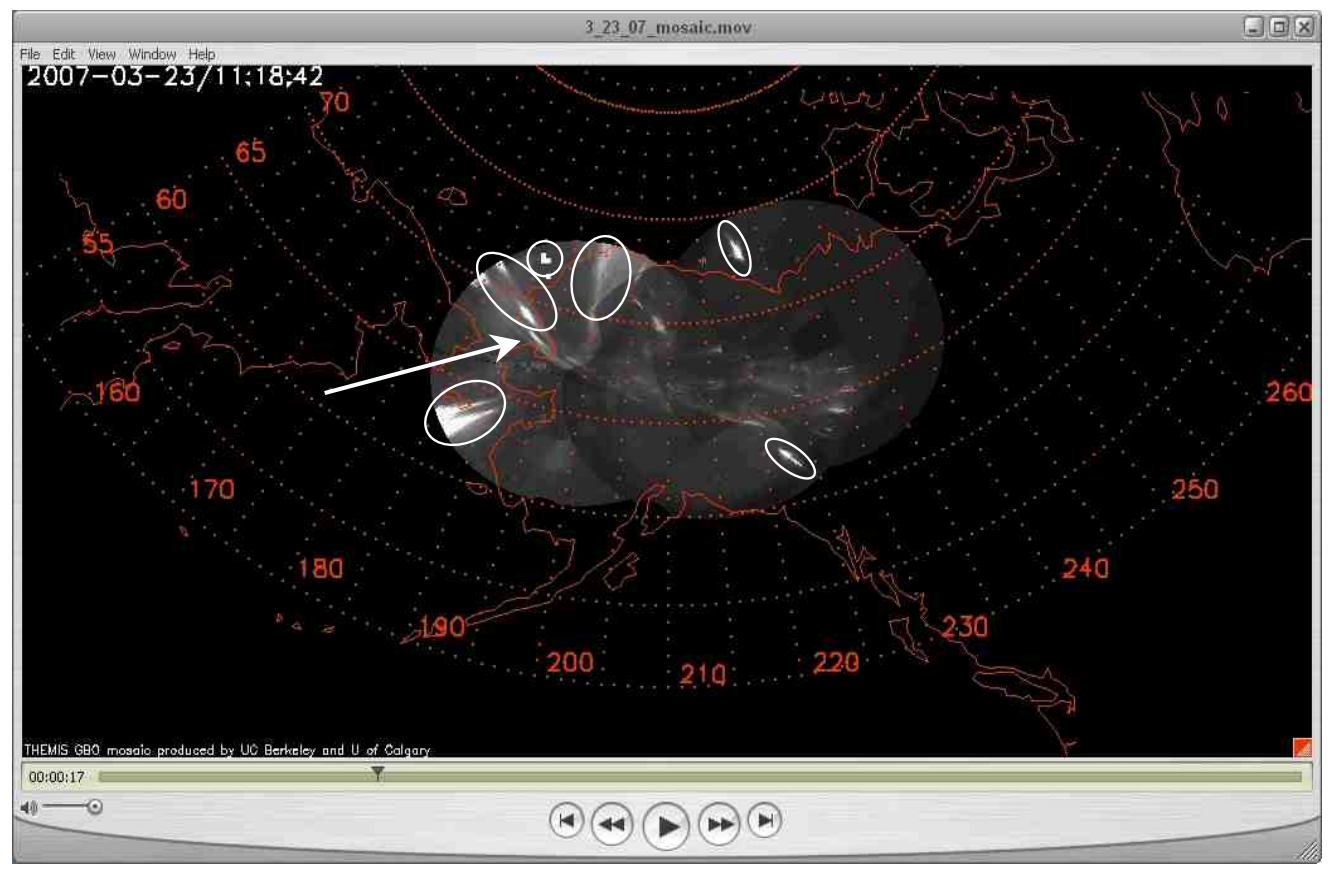

Figure 4. THEMIS GBO/ASI mosaic of auroral images from stations: Kiana, Fort Yukon and Inuvik, at 11:18:42 UT, i.e. at the time of the substorm main intensification, as determined by the Kiana ASI. Circles indicate non-geophysical emissions, mainly due to smoke from a factory near the Kiana station. (The smoke contamination of the station has since been fixed). The first indication of an auroral brightening over Kiana is shown with the arrow.

A comprehensive summary of the timing signatures on this event, using Pi2 pulsations from THEMIS and ancillary ground stations is presented by McPherron et al. (2008). Substorm timing, using PiB signals at high latitudes, is discussed by Rae et al. (2008). Further analysis of the ASI signatures is presented in Mende et al. (2008). Observations of field-aligned currents at the ionosphere and their relation to the THEMIS observations in space is presented in Strangeway et al. (2008). 
Mapping the THEMIS satellites with standard (average magnetic field) models presents a problem that is depicted in Figure 5. The left portion of the image shows standard mapping using the Tsyganenko 2001 (Tsyganenko 2002a, 2002b) model, by varying the level of geomagnetic activity (Dst, AE) to determine the range of the footpoint locations and hence get an estimate of the mapping uncertainty. According to this mapping, all THEMIS satellites have footpoints about $2 \mathrm{hrs}$ of MLT to the west of Alaska, and are well outside the westernmost location of the auroral activation at 21.5MLT. Considering that all satellites, such as TH-B in Figure 2, saw significant energetic particle flux increases, dipolarization, and flows, indicating that the spacecraft were within the meridian of the activity, the above mapping is suspect. One reason for this is the presence of a significant East-West interplanetary magnetic field that can penetrate inside the magnetosphere. Another reason is the distortion of the average magnetosphere during the late growth phase, which may be missing from the above average models. To represent those effects properly we relied on mapping using GGCM simulation model fields just prior to the onset occurring in the simulation. This is accomplished as described in Raeder et al. (2008). Specifically, in response to the realistic solar wind magnetic field, the simulation onset took place at 11:00UT, and thus fields just prior to it were used to map the THEMIS satellites. This mapping resulted in footpoints that were just to the west of Alaska, i.e., very near the substorm meridian, and is thus in better agreement with observations.
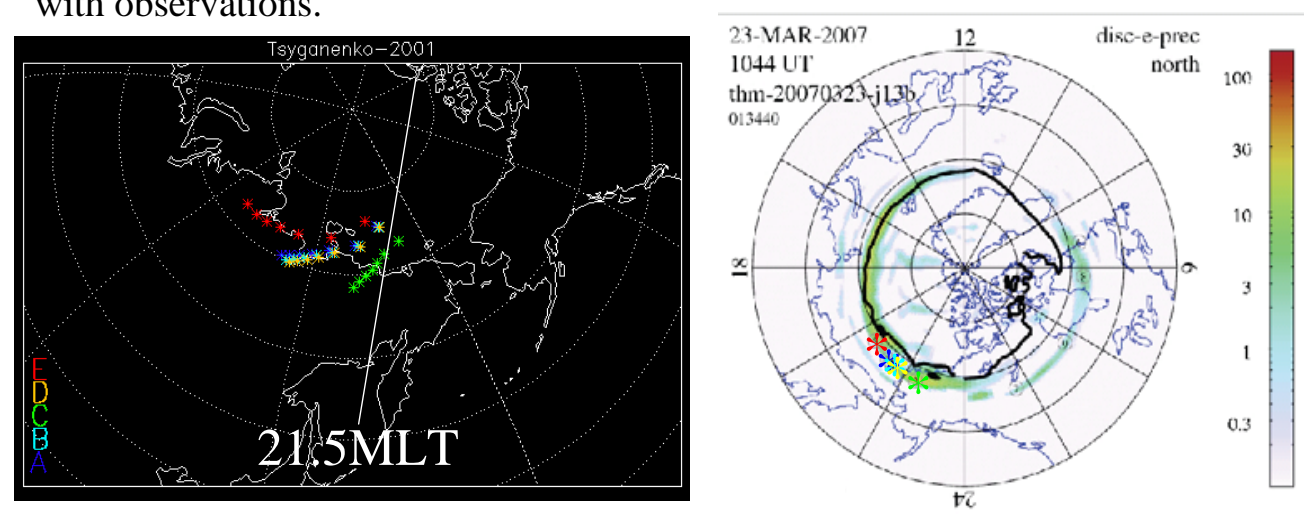

Figure 5. Field line mapping of the THEMIS satellites $C, D, B, A, E$ (from east to west, shown in Green, Yellow, Ciel, Blue and Red). Left: Mapping using the Tsynanenko 2001 model under various activity levels. Right: Mapping using realistic fields from GGCM simulation. The right model does a better job explaining the good relationship between the THEMIS measurements in space and the observed activity on the ground. 
Further evidence for a rapid evolution of the substorm current wedge comes from modeling of the ground magnetometer signals. Mid-latitude data from the intermagnet network were used in Figure 6 to fit model parameters of the ring current disturbance (DR), the partial ring current disturbance (DRP) and the substorm current wedge (SCW) to determine their magnitudes and locations as a function of time (Horning, 1976; Sergeev et al., 1996). The results show that starting around 11:14UT and until 11:18UT the substorm current wedge was localized around 01:30MLT and had a width of 1.5hr of MLT. This is more or less consistent with the images from POLAR/UVI, which showed onset at 01:00MLT. At 11:18UT the westward portion of the substorm current wedge expanded rapidly to -45 deg longitude within 2 minutes.
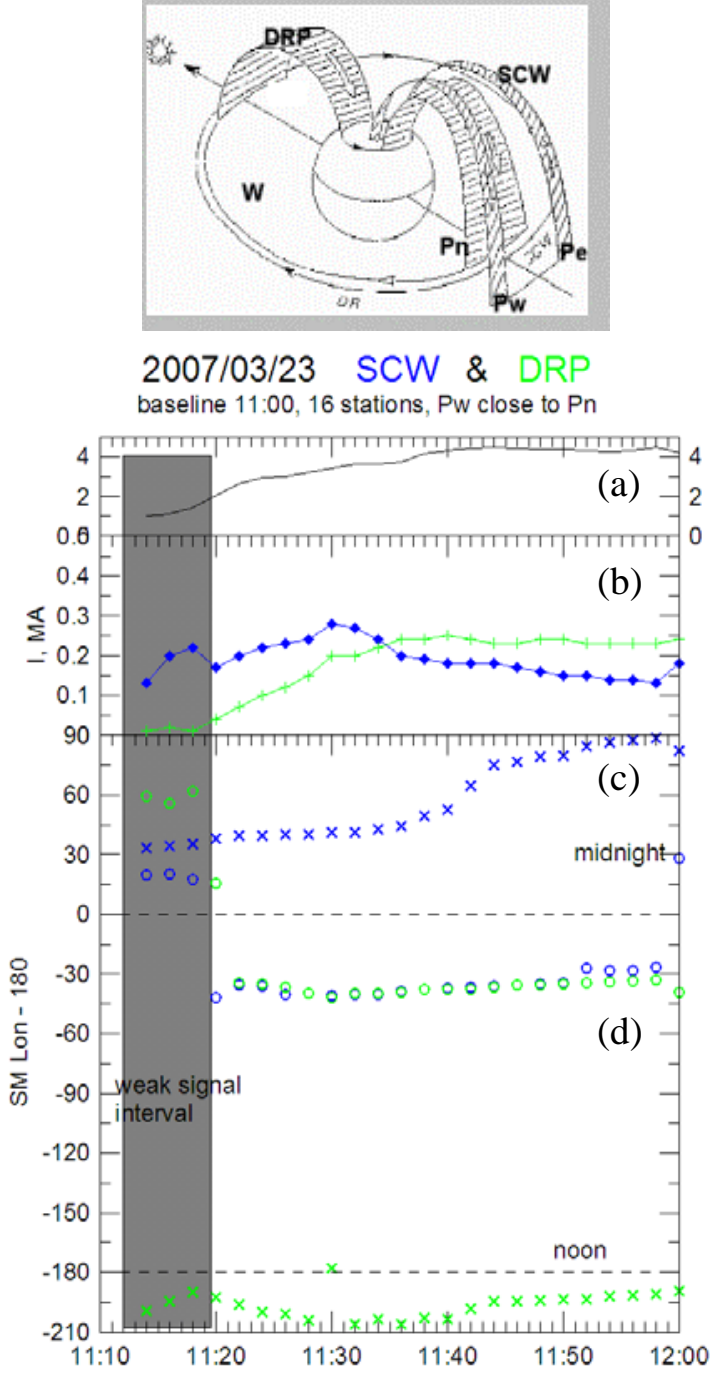

Figure 6. Modeling mid-latitude ground magnetometer signatures to determine the central meridian, width and intensity of near-Earth space currents. Top: Pictorial representation of the model used (composed of line currents or series of line currents). Bottom: Modeled intensity (MA) and longitude (degrees) of the current system components as a function of 
time past 11:10 UT, specifically: (a) standard deviation of observations versus model disturbances; (b) SCW (blue) and DRP (green) current intensity; (c) Longitude of downward (crosses) and upward (circles) fieldaligned currents, in SM-180 coordinates (i.e., Solar Magnetospheric longitude minus 180deg.).

As we have learned from the UVI images and the GBO/ASI images, the 11:18:45 UT intensification was an intensification distinct from the 11:10UT onset, and appeared approximately at 23:00MLT, i.e. at -15deg longitude, and expanded both eastward and westward. The above technique cannot distinguish between the longitudes of the main onset and its intensification. If, however, we assume the location of the new activation to be at $-15 \mathrm{deg}$, then the westward expansion speed of the SCW from the above analysis is about 2MLT hrs / 2min, consistent with the expansion observed in the UVI images.

When mapped geometrically in space at the location of THEMIS, the aforementioned westward expansion speed on the ground translates to a $280 \mathrm{~km} / \mathrm{s}$ azimuthal expansion at the location of TH-B (at a radius of $11.25 \mathrm{R}_{\mathrm{E}}$ ). Due to the flaring of the magnetic field towards the flanks, standard Tsyganenko model projections relate a pure azimuthal ionospheric motion (along a constant magnetic latitude), to a westward motion of an equatorial footpoint in space, with a nontrivial radial component of a variable direction. The radial component of the velocity depends on mapping model details and activity level. Figure 1 shows an example of a Tsyganenko (1989) model equatorial mapping points starting at $100 \mathrm{~km}$ in the ionosphere at 66deg magnetic latitude, and every 5deg in longitude, i.e., starting from 22MLT to the west, until 19MLT. Such an exercise suggests that a speed of $1 \mathrm{MLT} / \mathrm{min}$ in the ionosphere corresponds to a propagation speed of $300 \mathrm{~km} / \mathrm{s}, 350 \mathrm{~km} / \mathrm{s}$ and $200 \mathrm{~km} / \mathrm{s}$ predominantly in the $\mathrm{Y}_{\mathrm{GSM}}$ direction, and is around $250 \mathrm{~km} / \mathrm{s}$ between the location of TH-D,B,A and E satellites.

To determine if the projected speed matches the observed propagation speed in space we turn our attention to the THEMIS satellite observations. An expanded view of the data from TH-B around the time of the intensification is shown in Figure 7. It is evident that TH-B, south of the equator, measured a sharp dipolarization at around 19:30UT, accompanied by a reduction in field magnitude (which we interpret as entry into the high beta plasma sheet, closer to the neutral sheet) and a bipolar By signature superimposed on a permanent change in By which is evidence of a field-aligned current pair superimposed on a unidirectional current. These are classical signatures of plasma sheet recovery 
typically observed at late substorm expansion or substorm recovery (e.g. Pytte et al. 1976 JGR); however, in our case the satellites were already in the outer layers of a dense and hot plasma sheet.

One of the salient features important for understanding the buildup of the SCW is the fast Earthward flow pulse, seen almost simultaneously with the magnetic field depolarization, heating and slight density ramp-up and ramp-down. These are classical signatures of flow bursts, i.e., flow pulses observed within bursty bulk flow events (Angelopoulos et al. 1994; Sergeev et al. 1996). The flow burst is Earthward and slightly duskward and is superimposed on a long period flow wave (2.5min period). It is preceded by an energetic particle flux enhancement as seen in panels (d) and (e). Spin phase is equivalent to GSE longitude in panel (d). Particles moving at 120deg longitude are field-aligned (0deg pitch angle) and particles moving at-60deg are field- opposed (180deg pitch angle). Particles at +30deg have a 90deg pitch angle and a gyro-velocity that is duskward. The flux enhancement is seen first in the duskward moving particles. It is followed, about a half a minute later, by enhancements in the dawnward particle fluxes, which denote complete immersion of the satellite into the heated plasma sheet plasma (100keV gyro-centers on both sides of the satellite). It is evident from this panel that the hot plasma indeed expanded outward, i.e., southward, or lobe-ward over the satellite.

With the availability of more than one look directions and several energies it is possible to employ the technique of remote sensing (e.g. Daly 1984, Kettman and Daly 1988) to determine the orientation and speed of the approaching hot energetic particle boundary. The technique relies on the timing of the appearance of the hot fluxes at various gyro-center locations.

We looked at arrival times of particles at four specific energies $(40 \mathrm{keV}$, $100 \mathrm{keV}, 150 \mathrm{keV}$ and $300 \mathrm{keV}$ ). For simplicity we used only 90deg pitch angles (i.e. approximately -60deg and +30deg particle azimuths). Those particles are measured in eight gyro-velocity directions, obtained from the four SST detector mounting locations ( $\pm 52 \mathrm{deg}, \pm 25 \mathrm{deg}$ relative to the spin plane) times the two look directions possible normal to the magnetic field. The resultant gyro-velocity directions are $\delta= \pm 155, \pm 128, \pm 52, \pm 25$ measured from the $Y$-axis of an orthogonal right-handed coordinate system with $\mathrm{Z}$ that contains the spin direction and $\mathrm{X}$ along the field direction. Therefore, $\mathrm{X}$ is on the spin plane along the magnetic 
field azimuth, $\mathrm{Z}$ is along the spin axis along the ecliptic normal, and $\mathrm{Y}$ is 90 deg away from X, about 30 degrees away from Eastward. The resultant arrival times were modeled assuming a surface motion normal to the magnetic field of arbitrary orientation, $\varepsilon$, relative to the $\mathrm{Y}$-axis, and the variance chi-square $\left(\chi^{2}\right)$ of the data was plotted as a function of arrival times, to determine the orientation that minimizes that variance. The velocity of the boundary is then determined for that specific orientation.

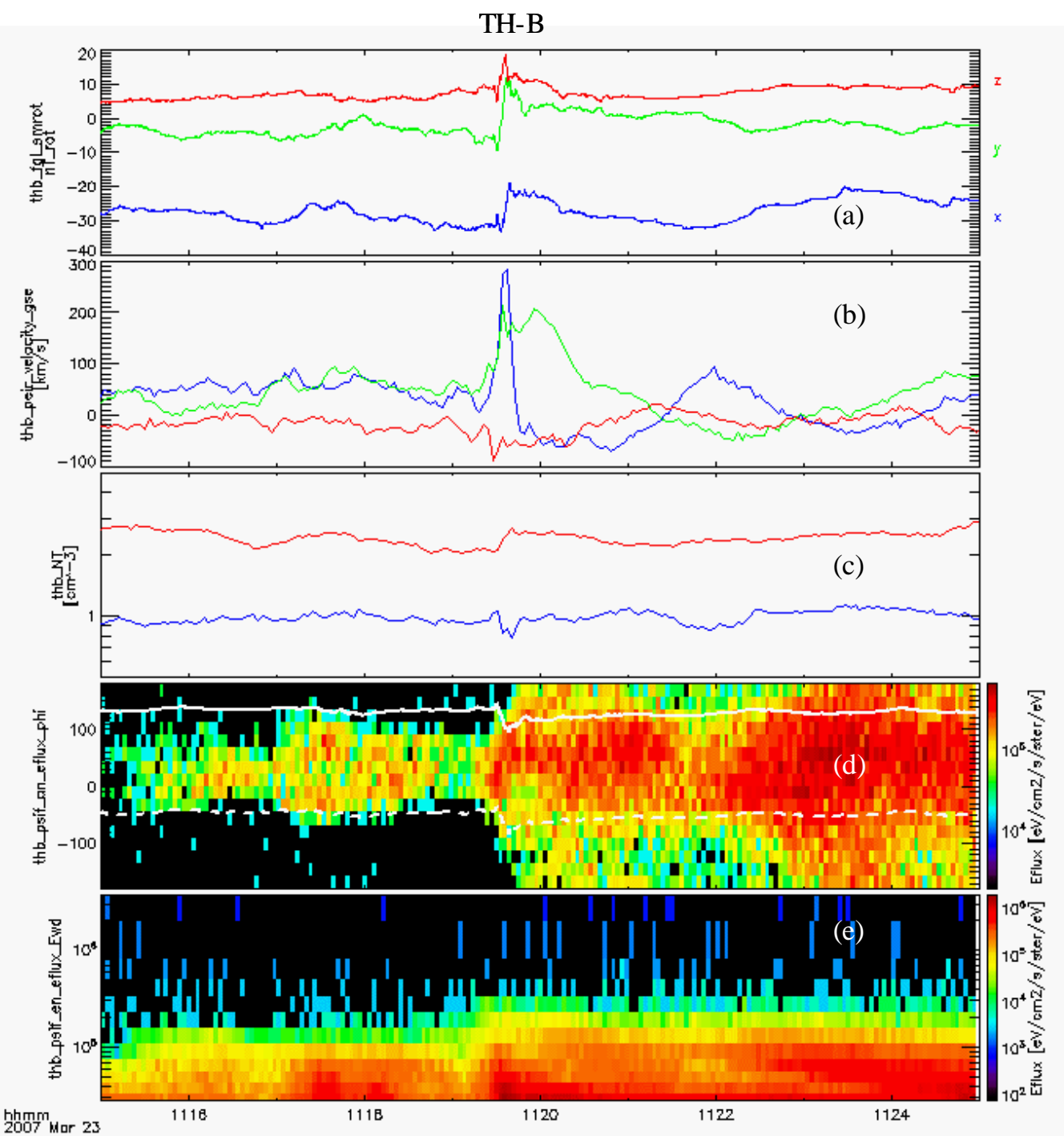

Figure 7. Detailed signatures of the substorm intensification on $\mathrm{TH}-\mathrm{B}$. (a) FGM measurements, 4S/s, SM coordinates, rotated about $Z_{S M}$ such that $X$ points towards Earth, and $Y$ azimuthally westward $(X, Y, Z$ correspond to blue, green and red); (b) ESA ion flow velocity in GSE coordinates; (c) Ion partial average temperature (red) and density (blue); (d) azimuth spectrogram of 100keV SST ion differential energy flux, with the magnetic 
field azimuth in the parallel and anti-parallel directions to the field line (solid and dashed white traces respectively); (e) energy spectrogram of Earthward SST ion differential energy flux (+/-45deg in azimuth and +/$43 d e g$ in elevation). The satellite spins with spin axis along ecliptic north; the magnetic field, pointing outward from Earth, is mostly on the spin plane (within 15deg) and therefore the particle azimuths relative to the magnetic field azimuth in panel (d) are a good approximation of a directional pitch angle - a positive or negative direction relative to the magnetic field, corresponding to the particle motion in gyrophase.

We used the singular value decomposition method (Press et al. 1989) for the least squares fit and obtained $\chi^{2}$ values shown, for probe B, in Figure 8. It is evident that the boundary orientation is well determined, since a deep minimum in chi squared is seen. The SST detector resolution in near-ecliptic elevations ( $\pm 25 \mathrm{deg}$ and $\pm 52 \mathrm{deg}$ ) bespeaks a boundary orientation accuracy that is on the order of 10deg or so.
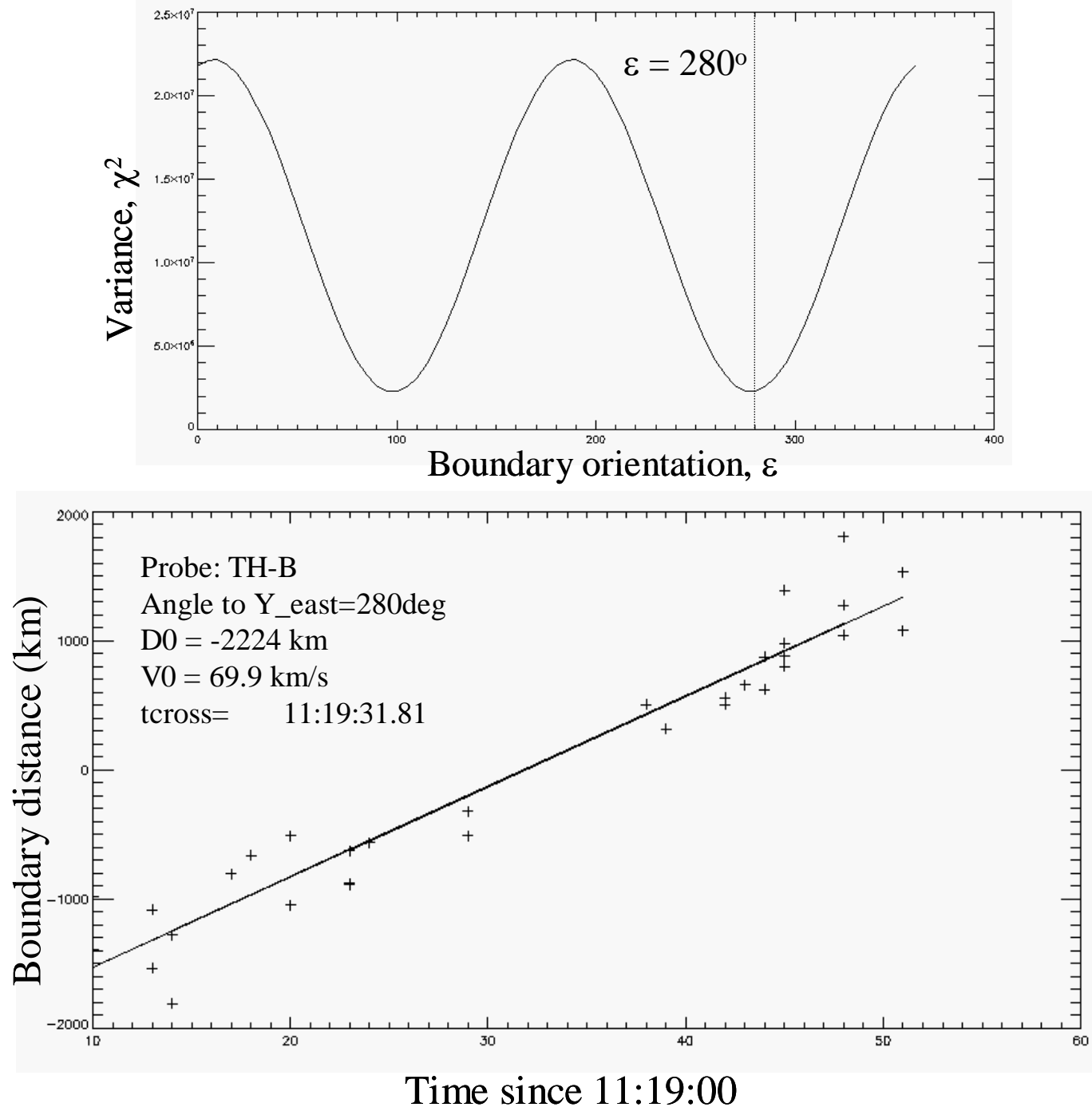

Figure 8. Remote sensing of the approaching plasma sheet hot plasma 
during the 11:18:42UT substorm onset on THEMIS satellite TH-B. The flux enhancement times of the $90^{\circ}$ pitch angle particles of various gyro-phases and energies was modeled assuming an approaching boundary at arbitrary orientation relative to the Eastward direction, to determine the orientation of minimum variance. That orientation was then used to determine the optimal boundary speed and boundary crossing time. Top: variance of the arrival times for various boundary orientations. Bottom: boundary distances corresponding to the timing measurements for appropriate gyro-radius, gyro-velocity direction and optimal boundary direction versus time (in seconds relative to 11:19:00UT). Negative or positive boundary distances correspond to an approaching or receding boundary respectively, assuming a positive speed.

The variance is minimized for a boundary angle of 280deg, which corresponds to a boundary velocity of $\sim 70 \mathrm{~km} / \mathrm{s}$, as is also shown in Figure 8 . Similar results were obtained from probes B and A and are tabulated in Table 1. They show that the motion of the active region at the outer layers of the plasma sheet where probes D, B and A were located, was lobe-ward and (possibly) slightly Eastward, in agreement with the convex shape of the plasma sheet surface near the flanks.

The differences between the arrival times of the boundary at the various spacecraft were $t_{D B}=4.2 \mathrm{~s}$ and $t_{B A}=6.6 s$. The distances of the satellites on $\mathrm{a}$ coordinate system that has $\mathrm{X}$ along the magnetic field and $\mathrm{Z}$ containing the spin axis is approximately $\mathrm{dZ}_{\mathrm{DB}}=420 \mathrm{~km}$ and $\mathrm{dZ}_{\mathrm{BA}}=600 \mathrm{~km}$, whereas if the magnetic field inclination is not accounted for in that distance (i.e. if the X-axis is along the magnetic field azimuth but on the spin plane) then $\mathrm{dZ}_{\mathrm{DB}}=220 \mathrm{~km}$ and $\mathrm{dZ} \mathrm{BA}_{\mathrm{BA}}=$ $330 \mathrm{~km}$. The resultant expansion speeds are consistent between the two pairs and vary with the above assumption between: $\mathrm{V}_{\mathrm{DB}} \sim \mathrm{V}_{\mathrm{BA}}=98 \mathrm{~km} / \mathrm{s}$ and $\mathrm{V}_{\mathrm{DB}} \sim \mathrm{V}_{\mathrm{BA}}=$ $49 \mathrm{~km} / \mathrm{s}$. These speeds are consistent with the expansion speed determined from remote sensing.

\begin{tabular}{|c|c|c|c|}
\hline & $\mathbf{t}_{\text {cross }}$ & $\mathbf{V}[\mathbf{k m} / \mathbf{s}]$ & $\boldsymbol{\varepsilon}[\mathbf{d e g}]$ \\
\hline D & $11: 19: 27.6$ & 75 & 270 \\
\hline B & $11: 19: 31.8$ & 70 & 280 \\
\hline A & $11: 19: 38.4$ & 80 & 275 \\
\hline
\end{tabular}

Table 1. Results of remote sensing analysis on the inner probes

Timing of the arrivals of the other signatures at the inner three spacecraft is also consistent with the expansion of the heated plasma from one probe to another. Figure 9 shows a summary of the time series flow velocity, magnetic field and energetic particle flux measurements on TH-D, B, A and E. The timing 
from the remote sensing technique is denoted in dashed lines. The results are consolidated in Table 2.

TH-E, further to the west, was further away from the nominal neutral sheet, but very close to the actual neutral sheet, as evidenced by the sign changes in Bx prior to the occurrence of the fast flow. Due to TH-E's proximity to the neutral sheet, TH-E maps onto the equatorial plane locally, contrary to TH-D, B, and A, whose equatorial footpoints likely map much further downtail. The energetic particles, which were measured at the location of TH-D, B and A at 11:19:30 UT and were likely injected near the neutral sheet at an earlier time, travel westward and were seen as a flux enhancement at 11:19:20 UT at TH-E, about 30 seconds prior to the arrival of the flow burst and the dipolarization there. The flux at all energies (not shown) increased gradually at TH-E, with no sharp injection, preventing the opportunity to apply remote sensing of an expanding boundary there. At the same time By perturbations prior to the dipolarization at TH-B complicate detection of a sharp field-aligned current boundary there. However, both dipolarization and fast flow were detected at TH-E and their timing is included in Table 2.

Timing of all signatures on TH-D, B and A consistently points toward a picture of outward local plasma sheet expansion, encompassing flows, depolarization and field-aligned current sheets. The activity boundary is layered horizontally. However, timing of the flow and dipolarization arrival to TH-E suggests a different process. The D-E spacecraft separation along $\mathrm{Y}$ is $\sim 2.5 \mathrm{R}_{\mathrm{E}}$ and the propagation delay using the timing tabulated in Table 2 is $\sim 250 \mathrm{~km} / \mathrm{s}$ (we used TH-D since of the three, TH-D, B and A, TH-D is closest to the neutral sheet). This speed is much faster than the speed of the lobeward expansion of the boundary. Our interpretation of the rapid Westward evolution of the Earthward flow burst and dipolarization is that these phenomena are mapped to the westward traveling surge observed expanding westward on the ground. The observations of TH-D, B, A demonstrate the link between the evolving Earthward flow channel, the field-aligned currents and particle injections. The phenomena may have started earlier near the neutral sheet, but not seen at its outer layers where the D, B, A probes were located. Thus the time delay between TH-D and TH-E may be an upper bound on the expansion velocity. Nevertheless, that velocity is comparable 
to the expected expansion velocity from ground observations and our simple mapping arguments.

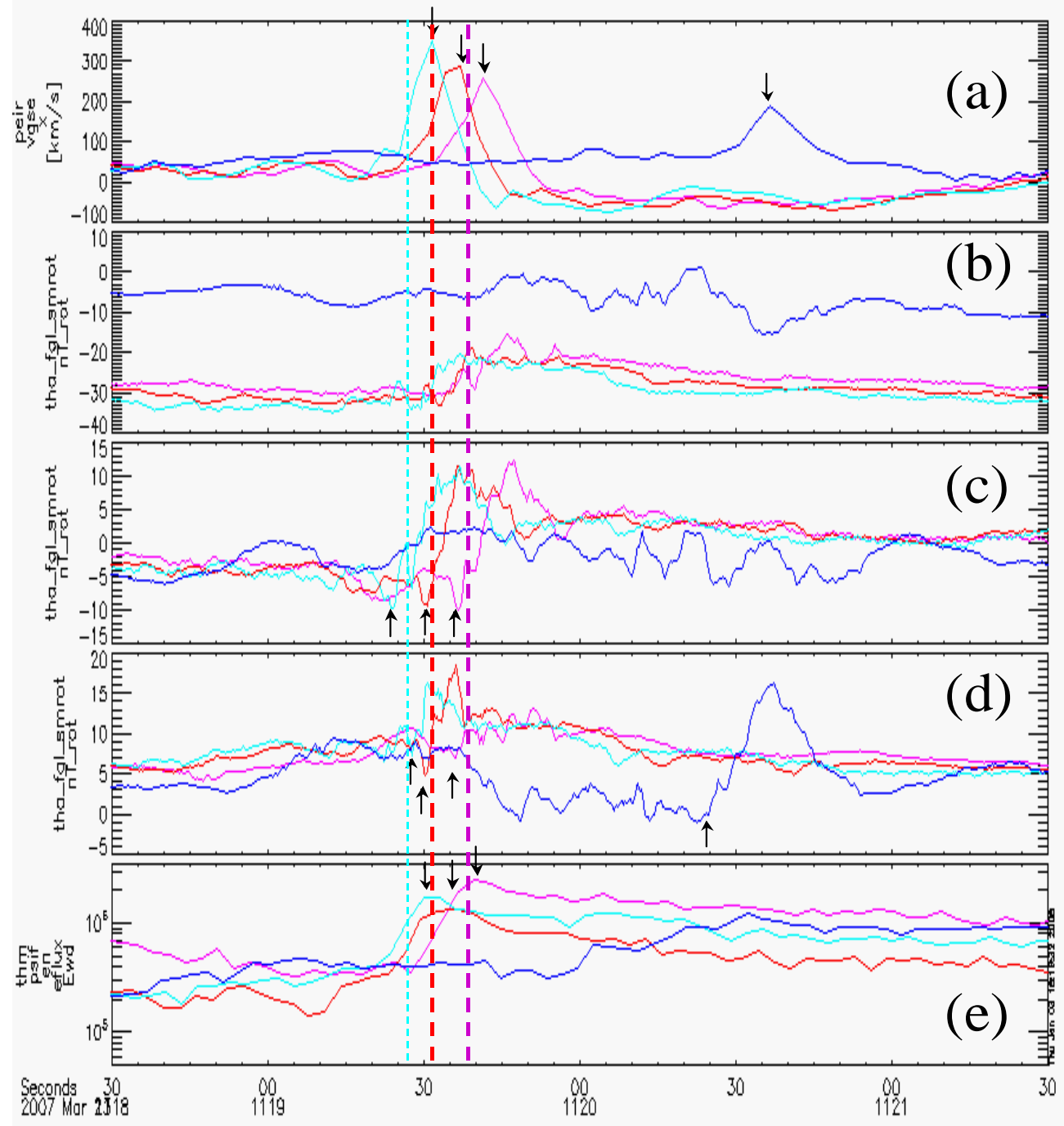

Figure 9. Timing of particles and fields signatures at the various spacecraft. THD, B, A and E are shown in Ciel, Red, Magenta and Blue, respectively, per the standard THEMIS color scheme, as in Figure 1. The times are also tabulated in Table 2. (a) $X_{G S E}$ component of the flow velocity from the ESA instrument; (b), (c) and (d) $X, Y, Z$ components of the magnetic field, in 'rotated' Solar Magnetospheric coordinates, where the $X$ and $Y$ axes have been rotated about $Z_{S M}$ coordinate, such that $X$ points towards Earth and $Y$ is azimuthally westward; (e) the differential energy flux from the $40 \mathrm{keV}$ channel of the SST ions. TH-E was near the neutral sheet at the time, and saw no sharp signature in $B_{y}$ and in energetic particles, but did experience the fast flow and the associated dipolarization. 
In summary, using THEMIS observations of a substorm on March 23, 2007, at 11:10UT, we have shown that the westward traveling surge propagates on the ground at a speed that is commensurate with the expansion of the substorm current wedge in space. The speed of the expansion was observed to be $\sim 1 \mathrm{MLT} / \mathrm{min}$, both on the ground and in space. This speed is much faster than could have been substantiated using the widely separated GOES satellites, but consistent with expectations from statistical results by Nagai $(1982,1991)$ that the expansion speed can be quite large when observed close to the activation meridian. To our knowledge these are the first clear simultaneous observations of the expansion of the westward traveling surge on the ground and in space. They show that the westward expansion of the substorm current wedge is due to the impulsive buildup of dipolarized flux, accompanied by Earthward flow bursts.

Further analysis of the timing on this event can be found in Liu et al. (2008). Further analysis of the low frequency compressional oscillations and particle perturbations can be found in Keiling et al. (2008). The variability of the inner edge of the plasma sheet is further expanded upon in Runov et al. (2008).

\begin{tabular}{|c|c|c|c|c|c|c|c|}
\hline & $\mathbf{D}$ & $\mathbf{B}$ & $\mathbf{A}$ & $\mathbf{E}$ & $\mathbf{t}_{\mathbf{D B}}$ [sec] & $\mathbf{t}_{\mathbf{B A}}$ [sec] & $\mathbf{t}_{\mathbf{D E}}$ [sec] \\
\hline $\mathbf{V x}$ & $19: 31$ & $19: 36$ & $19: 41$ & $20: 36$ & 5 & 5 & 65 \\
\hline $\mathbf{B y}$ & $19: 24$ & $19: 30$ & $19: 36$ & $\mathrm{n} / \mathrm{a}$ & 6 & 6 & $\mathrm{n} / \mathrm{a}$ \\
\hline $\mathbf{B z}$ & $19: 22$ & $19: 30$ & $19: 36$ & $20: 23$ & 8 & 6 & 61 \\
\hline $\mathbf{S S T}_{\text {eflux }}$ & $19: 29$ & $19: 35$ & $19: 42$ & $\mathrm{n} / \mathrm{a}$ & 6 & 7 & $\mathrm{n} / \mathrm{a}$ \\
\hline $\mathbf{R S}$ & $19: 27.6$ & $19: 31.8$ & $19: 38.4$ & $\mathrm{n} / \mathrm{a}$ & 4.2 & 6.6 & $\mathrm{n} / \mathrm{a}$ \\
\hline
\end{tabular}

Table 2. Summary of timing results (mm:ss) on probes $T H-D, B, A$ and $E$

When the plasma sheet is observed further from the neutral sheet, close to or at the plasma sheet boundary, the flow burst, magnetic dipolarization and the heated plasma are observed to move lobe-ward, consistent with a local expansion of the plasma sheet. A single satellite is sufficient to determine the outward expansion speed and boundary orientation of the heated plasma. This is proposed for the primary phase of the THEMIS mission, when the inner satellites $(\mathrm{P} 3,4,5)$ will be located at lower inclinations within the plasma sheet, i.e., nominally within $<2 \mathrm{R}_{\mathrm{E}}$ from the neutral sheet. The lateral motion of a planar surface can then be modeled as a field-aligned current, and the spatial extent, latitudinal width and magnitude of that current can be inferred. When independent measurements of the field-aligned current are made using magnetometer data at appropriately positioned spacecraft separations, the planar assumption can be further validated, 
and--using high time resolution magnetometer measurements-- the microstructure of these currents, including their spatial scales, can be determined and placed in a global context. This will be tested out at $1 \mathrm{R}_{\mathrm{E}}$ separation scales during the secondyear nominal mission by taking advantage of the cross-sheet separation of P5 and P3/P4 satellites. It is well known that the westward traveling surge is composed of multiple current systems that map to scales smaller than $1 \mathrm{R}_{\mathrm{E}}$ to the equatorial magnetosphere (Hoffman et al 1994). Plans for a more comprehensive study of smaller scale current filaments within the substorm current wedge will have to await an extended mission, with a tighter clustering of the inner THEMIS probes.

\section{THEMIS observations at the day side}

THEMIS traversed the day side magnetosphere in a string-of-pearls configuration, which allowed it to determine the extent and motion of flux transfer events (FTEs, Russell and Elphic, 1979) and dissect their structure. Figure 10 shows the spacecraft locations for such an encounter on July 12, 2007. During this inbound crossing, TH-A, the trailing probe, was in the magnetopause. Its data are shown in Figure 11.

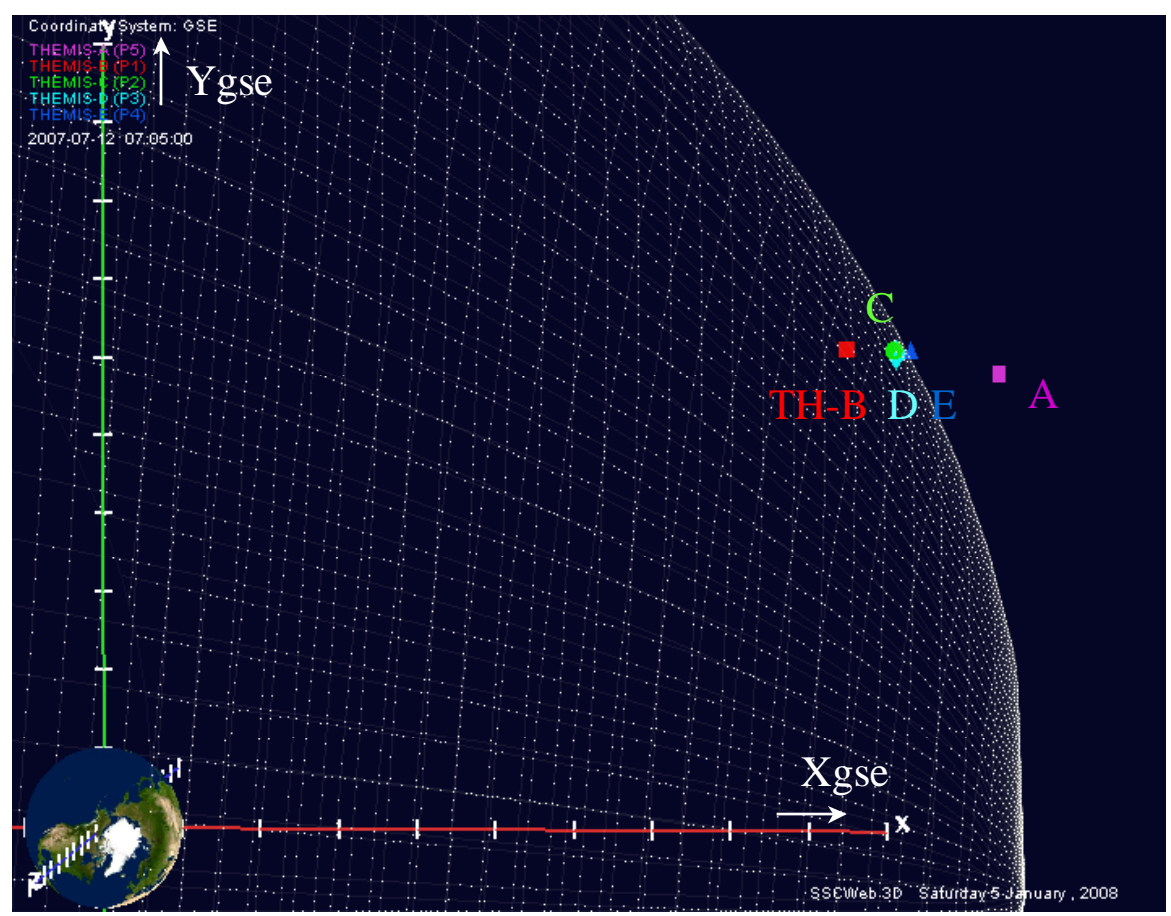




\begin{tabular}{|l|r|r|r|r|}
\hline \multicolumn{1}{|c|}{ Satellite } & Color & \multicolumn{1}{c|}{$X$} & \multicolumn{1}{c|}{$Y$} & \multicolumn{1}{c|}{$Z$} \\
\cline { 2 - 5 } THEMIS-A (P5) & & 11.114 & 5.582 & -3.39 \\
\hline THEMIS-B (P1) & & 9.214 & 5.928 & -2.94 \\
\hline THEMIS-C (P2) & & 9.803 & 5.899 & -3.101 \\
\hline THEMIS-D (P3) & & 9.827 & 5.849 & -3.093 \\
\hline THEMIS-E (P4) & & 10.011 & 5.907 & -3.174 \\
\hline
\end{tabular}

Figure 10. THEMIS probe locations [GSE, $R_{E}$ ] on July 12, 2007, 07:05UT. Satellite locations are also tabulated in GSE coordinates. Magnetopause location for a solar wind dynamic pressure of $1.3 \mathrm{nPa}$ is also shown, consistent with the observed location.

The magnetic field and velocity are in the magnetopause coordinates (LMN), where $\mathrm{N}$ is normal to a model magnetopause (Shue et al. 1998), with solar wind parameters obtained from WIND; $\mathrm{L}$ is normal to $\mathrm{N}$ containing the model field inside the magnetosphere (roughly Northward), and M is orthogonal to the other two directions; in our case predominantly duskward. The bipolar signature of a transient encounter with an FTE is evident at 07:04 UT in $\mathrm{B}_{\mathrm{N}}$ (red), whereas $\mathrm{B}_{\mathrm{L}}$ shows that the core of the FTE was pointing mostly southward.

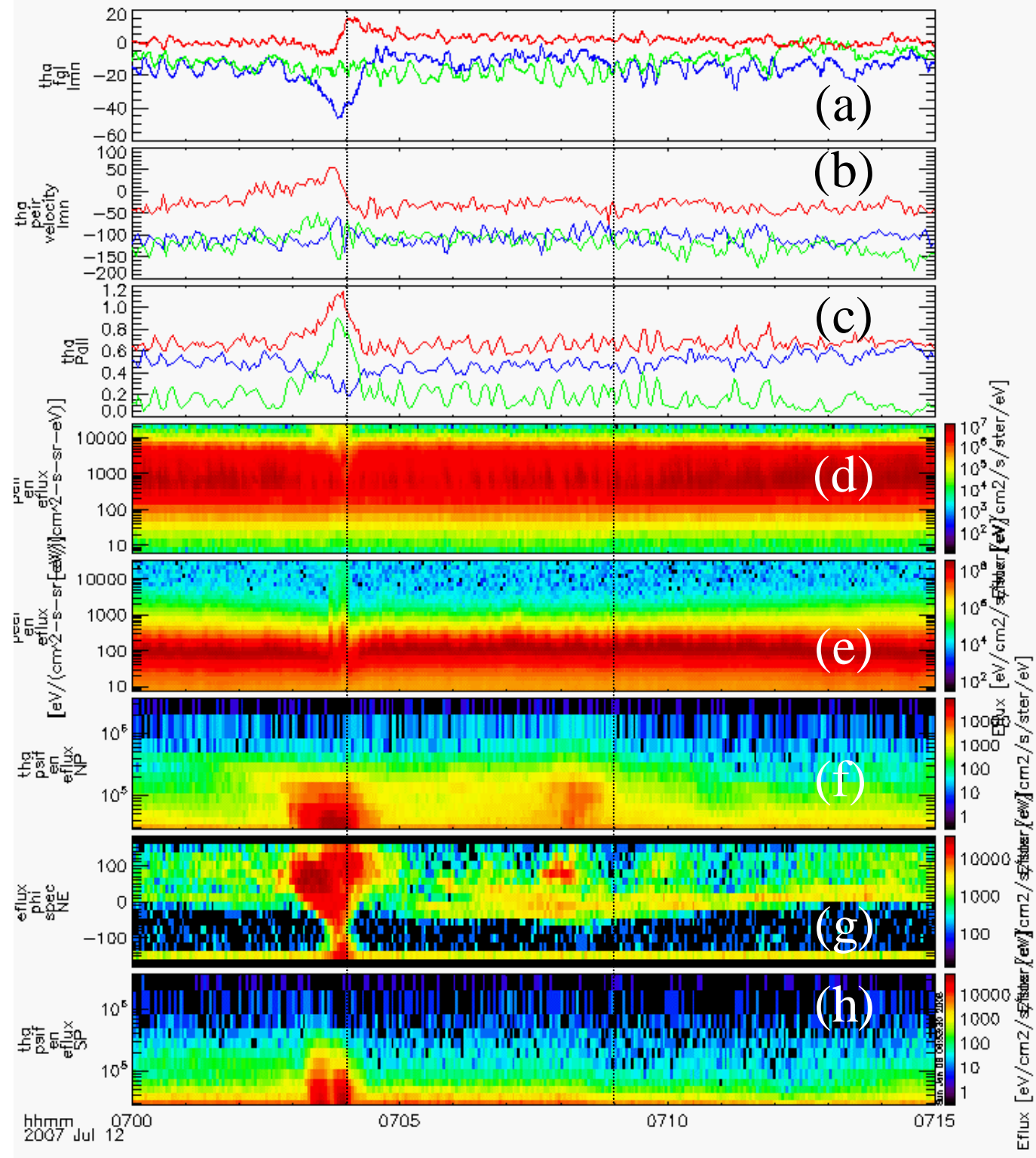


Figure 11. TH-A observations July 12, 2007, 07:00-07:15UT. The dashed vertical lines indicate FTE observations on TH-C (in Figure 13). (a) Magnetic field [nT] in $L M N$ coordinates: $L, M, N$ are blue, green and red lines; (b) Partial velocity [km/s] from the ESA instrument in LMN coordinates; (c) Magnetic (blue), plasma (green) and total (red) pressure [ $n P a]$; (d) ESA ion omnidirectional differential energy flux [eV/(cm2 s str eV)] (a.k.a. eflux); (e) Same as (d) but for ESA electrons; (f) energy spectrogram of the SST ion eflux from the North-Polar (NP) detector, looking at 52deg above the spin plane; (g) angular (azimuth) spectrogram of SST ion eflux from the North Equatorial detector, looking at 25deg above the spin plane; (h) energy spectrogram of the SST ion eflux from the South-Polar (SP) detector, looking at 52deg below the spin plane. It is evident that the bipolar signature on TH-A (this plot) was centered slightly earlier (about 10s) than the dashed line at 07:04UT. The dashed line at 0709UT was an FTE observed on the other probes but only remotely sensed in TH-A energetic particles due to their finite gyroradius.

As the FTE was approaching the spacecraft, the boundary moved outward and then inward, as evidenced in the bipolar signature in $\mathrm{V}_{\mathrm{N}}$. The ESA ion and electron spectra show a transient crossing of the boundary layer, lasting about 20s. The total plasma and magnetic pressure show a peak at the center of the FTE, which is evidence of the tension forces within the FTE due to the field line curvature there.

At the time prior to the FTE encounter, the magnetic field angle was 60deg below the spin plane and +120 deg in azimuth, i.e., anti-Sunward and roughly tangent to the magnetopause. After the FTE passage the field direction retained the same azimuth, but the elevation changed to about 20deg below the spin plane. In either case, the energetic particle data from the SST particles moving in the North Polar (NP) direction (i.e., measured by the detector looking in the southpolar direction). The particle velocities, centered at 52deg above the spin plane, have roughly $90^{\circ}$ pitch angles, with gyro-centers that were on the Earthward side of the spacecraft. The energy spectra of the NP particles show clearly the arrival of the FTE ahead of its magnetic signature, remotely sensing its arrival due to the finite gyroradius effect of the energetic particles. Higher energies were detected first, as they correspond to larger gyro-radii. The dispersion is clear in the case of the 07:04 UT FTE. Similar energy dispersion signatures are seen at 07:09 UT, but there is no accompanying magnetic signature of an FTE at that time at TH-A.

The SST particles moving in the North-Equatorial direction correspond to a larger solid angle portion of the nearly field-aligned and nearly field-opposed particles, and thus a wider range of pitch-angles at that time. That direction was 
partially affected by sunlight at the time, as evidenced by a horizontal bar at nearly anti-sunward particle directions; this was expected because the open detector is looking straight into the sun during a portion of the spin. The quick recovery of the electronics, which salvages most look directions, bespeaks a nominally operating instrument. The angular spectrogram of that detector is shown in Figure 11, panel g. It is evident that for several minutes prior to the FTE arrival, the SST detector was measuring higher flux of $90^{\circ}$ spin phase particles, coming from the magnetosphere. Just prior to the arrival of the FTE the 90deg pitch angles increased in flux, and isotropized only inside the FTE. The SST particles moving in the South-Polar direction, 52deg below the spin plane, correspond to either field-aligned or $90^{\circ}$ pitch angles of gyro-centers that are predominantly on the sunward side of the spacecraft. The energy spectrum of the South-Polar moving particles is shown in the panel below. It is evident that the particle fluxes there are much reduced relative to North-Polar moving particles.

From the time delay of $55 \mathrm{~s}$ in this isotropization and from the gyroradius of $100 \mathrm{keV}$ particles in the observed 28nT field, we compute a speed of $50 \mathrm{~km} / \mathrm{s}$ for this FTE boundary entry. This is smaller than the observed flow speed, but is expected since the encounter of the FTE happens at an oblique angle to the flow. The opposite dispersion is observed, on a similar time scale, upon exit from the FTE energetic particle boundary. Similar remote sensing signatures are evident in the angular spectrogram of ( $g$ ) on either side of the dashed line centered at 07:09UT, indicating similar effects on the second, remotely sensed, FTE.

The data from probe TH-E, TH-C and TH-B are shown in Figures 12, 13 and 14, in that order, which represent their decreasing distance from Earth. TH-D was very close to TH-C and observed similar signatures as TH-C (at spinresolution), though interesting differences appear in high-resolution fields data.

TH-E and TH-C were in the magnetosheath proper at 07:00UT and entered the magnetopause boundary layer a couple of minutes later as evidenced by the electron and ion spectra which show enhancement in the $10-20 \mathrm{keV}$ particles. TH-E and TH-C crossed of the magnetopause current layer about a minute later. Both boundary layer and magnetopause transitions were seen first on TH-C, closer to the magnetosphere, and then on TH-E, as expected for an outward motion of the magnetopause boundary. TH-E exited the current layer at 07:09:30UT, followed by TH-C at 07:12:30UT, i.e., in reverse order. After that 
the field at the two probes was in the same direction and magnitude as in the sheath (as monitored on TH-A, in Figure 11). The flow within the magnetopause boundary layer was in the same average direction as in the sheath, but much more variable.

TH-C observed clear bipolar signatures of two FTEs, as determined by the bipolar signatures in $\mathrm{B}_{\mathrm{N}}$. The FTEs were centered at 07:04UT and 07:09UT.

TH-B was in the magnetosphere at 07:00UT and encountered the magnetopause boundary layer between 07:04:20 and 07:05:40 UT, as evidenced by the higher flux particle spectra there, with ions peaking in energy at $1 \mathrm{keV}$ and electrons at around $100 \mathrm{eV}$. TH-B entered and exited the FTE current layer at 07:04:15-07:04:30 UT (as evidenced by the $\mathrm{B}_{\mathrm{N}}, \mathrm{B}_{\mathrm{M}}$ values which match the data during the FTE encounters on TH-E and C), without traversing the magnetopause current layers, as shown by the fact that $\mathrm{B}_{\mathrm{M}}$ never became negative, i.e., the field never attained a southward direction as in the magnetosheath.

Based on ion and electron spectra we can determine that TH-E remained in the boundary layer throughout most of the rest of the interval surrounding the FTEs, except for a short interval at 07:06- 07:06:30 UT. Thus TH-E encountered the two FTEs from within the magnetopause boundary layer, though from the magnetospheric side of the current layer. TH-C entered the magnetosphere at 07:02:30 UT and again at 07:05:30 UT; in both cases it encountered the FTEs starting from the magnetospheric side, and entered/exited the FTE magnetic structure and surrounding magnetopause boundary layer particles due to the passage of the FTEs. Like TH-C, TH-B was at the magnetospheric side prior to the FTE encounter, and observed the first FTE particle and current layers, but did not observe the second FTE particle or current layer. Thus TH-C only remotely sensed the 07:09UT FTE signatures from the magnetospheric side proper. 


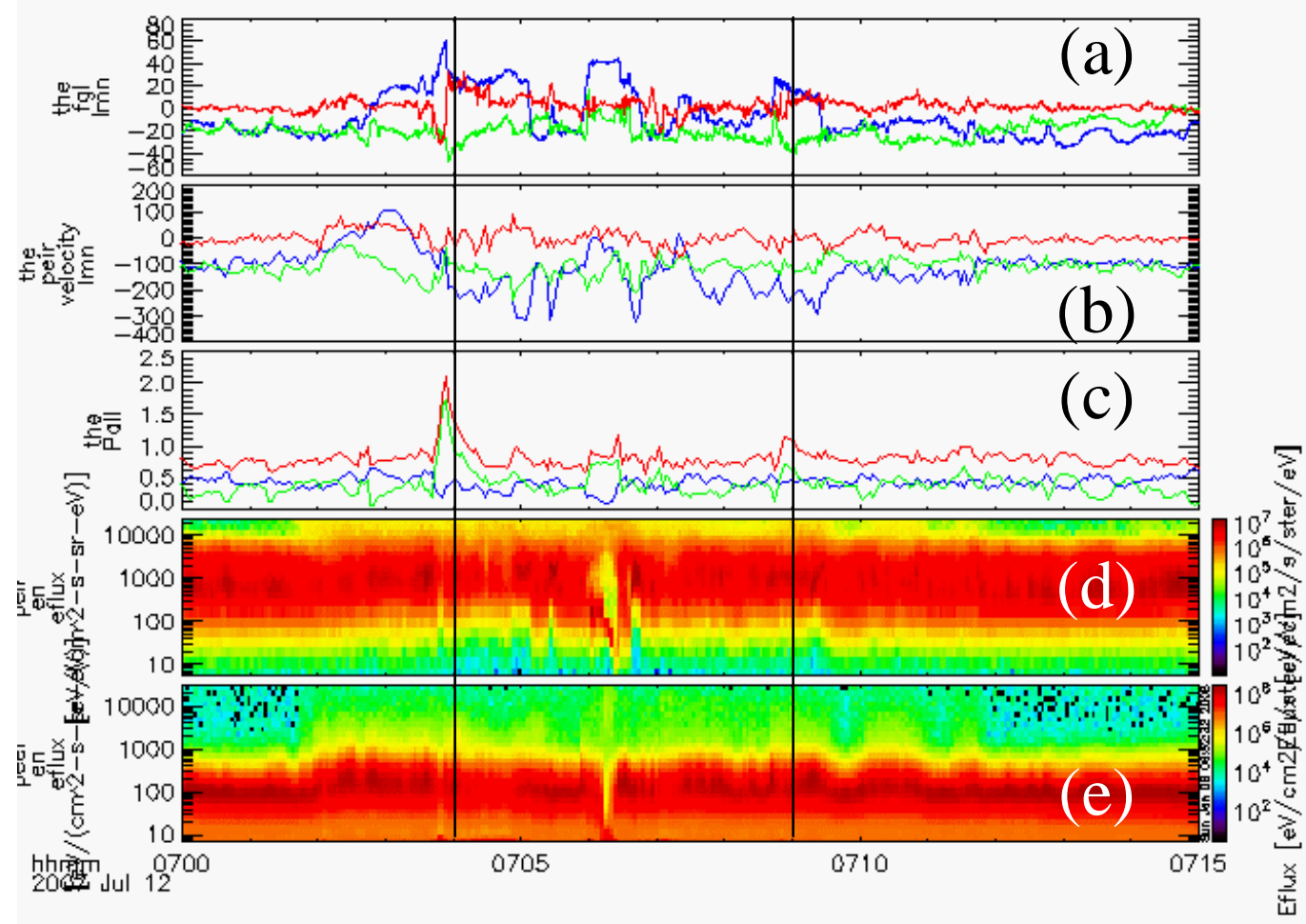

Figure 12. TH-E data in identical format as Figure 11. (a) Magnetic field [nT, LMN: blue, green, red]; (b) Partial ESA velocity [km/s, LMN]; (c) Magnetic (blue), plasma (green) and total (red) pressure [ $\mathrm{nPa}$; $(\mathrm{d}) \&(e)$ ESA ion \& electron omnidirectional e flux [eV/(cm2 s str eV)]. Dashed lines denote, again, timing of FTE centers on $\mathrm{TH}-\mathrm{C}$, observed slightly earlier on TH-E.

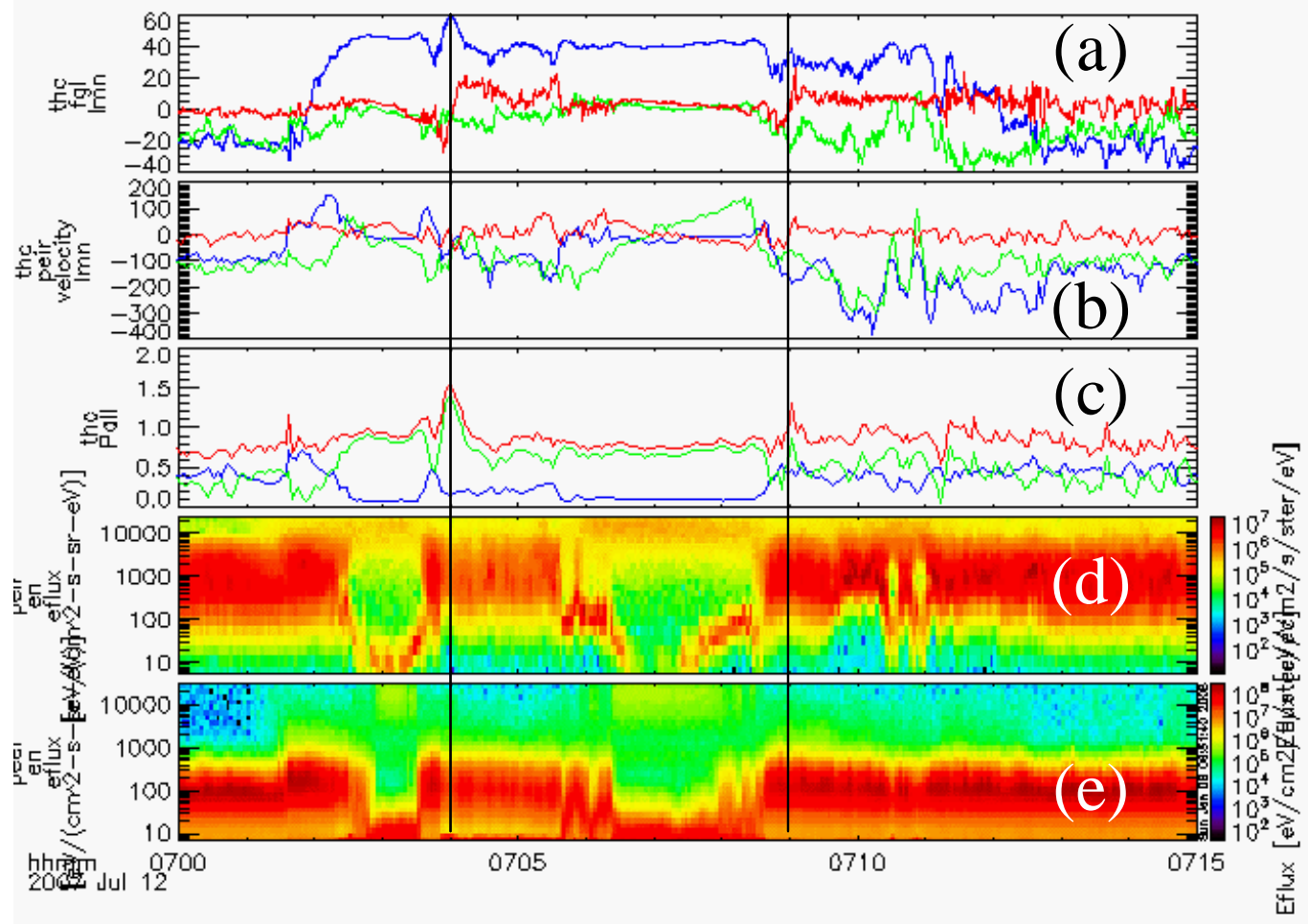

Figure 13. $\mathrm{TH}-\mathrm{C}$ data, in identical format as Figure 12. Dashed lines indicate the center of the FTE (center of bipolar signature in $B_{N}$ ). 


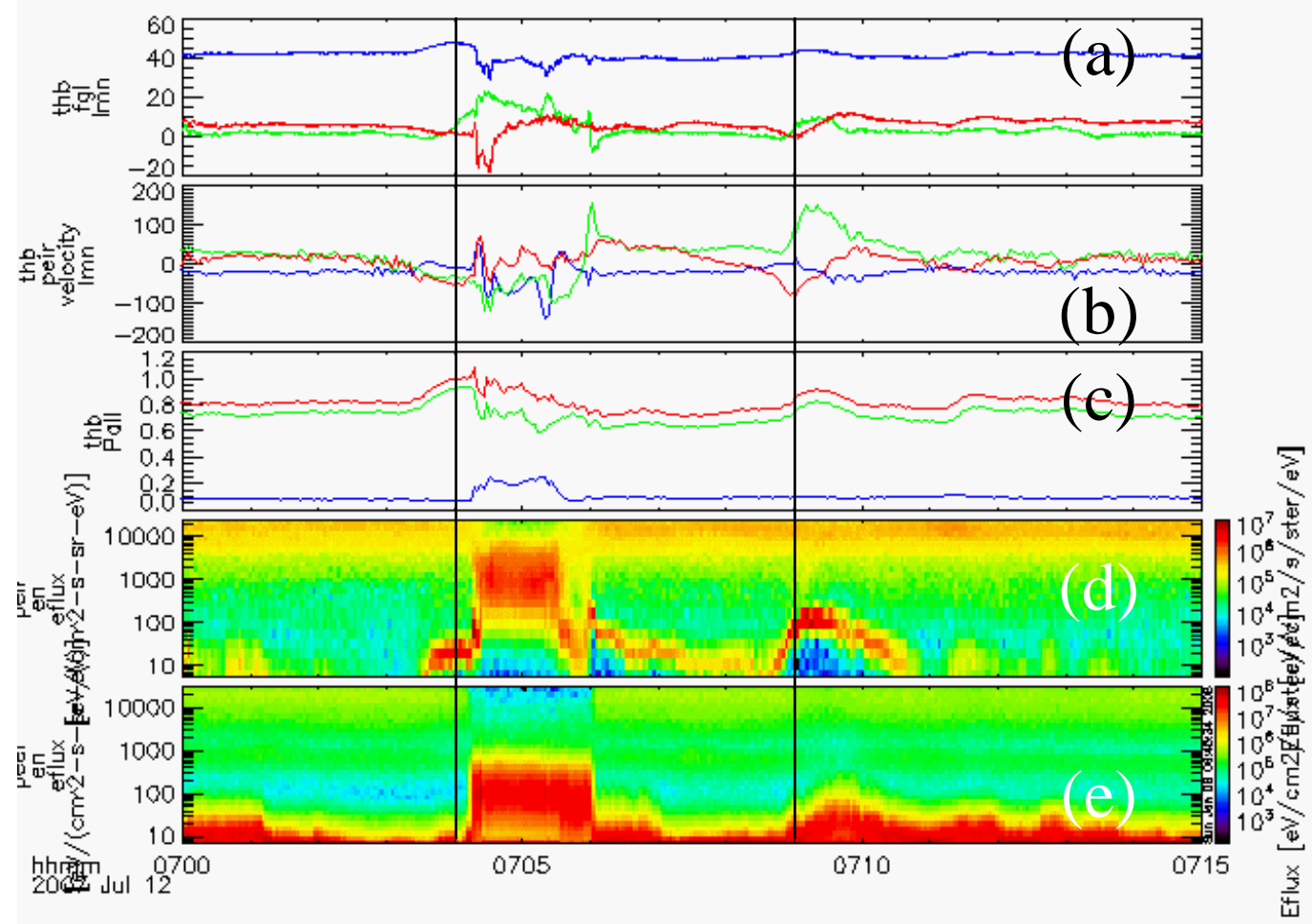

Figure 14. TH-B data, in identical format as Figure 12. Dashed lines indicate the center of the FTE on TH-C (center of bipolar signature in $B_{N}$ ). On TH-B, both FTES were encountered slightly later than on TH-C.

The low energy, cold particle signatures observed in the ion spectra inside the magnetopause (TH-C, B) are cold plasmaspheric plume ions, which are typically observed in the post-noon sector on THEMIS and are described in greater detail in McFadden et al. (2008). Their characteristic rise and fall in energy is related to increases in the plasma flow velocity, which enables this cold and otherwise nearly stationary population to ExB drift with the rest of the plasma populations into an energy where it is measurable by the ESA instrument (i.e., above the lower energy cut-off at $\sim 5 \mathrm{eV}$ ).

The time delays of the FTE observations between TH-A, C and E are consistent with a lateral motion of the FTE past those spacecraft. However the time delays must be obtained after careful modeling to determine FTE orientation and structure, which is beyond the scope of this paper. Differences between the FTE motion and the magnetopause boundary layer motion are important in determining whether the FTE is a structure advected passively by the ambient flow, or an evolving magnetic structure that is moving relative to the ambient boundary layer plasma, subject to active reconnection forces.

That this event is an excellent "in vivo" FTE event is evident in the flow velocities, which are seen (on all probes) to deviate considerably from the 
magnetosheath flow around the FTE encounter. Monitoring of the steady magnetosheath flow on TH-A reassures us that these variations are not solar-wind related. Specifically, bipolar $\mathrm{V}_{\mathrm{M}}$ and $\mathrm{V}_{\mathrm{L}}$ flows are seen on TH-E and TH-D to reach or exceed $300 \mathrm{~km} / \mathrm{s}$, compared to a magnetosheath flow of $180 \mathrm{~km} / \mathrm{s}$.

Observations of the boundary normal flow velocity, $\mathrm{V}_{\mathrm{M}}$, reveal that the plasma motion is consistent with an approaching or retreating bulge in the magnetopause on both sides. This signature is cleanest when the satellite is outside the magnetopause layer prior to the encounter. For example, on the 07:04UT FTE, TH-A observed an away $\left(\mathrm{V}_{\mathrm{N}}>0\right)$ then towards $\left(\mathrm{V}_{\mathrm{N}}<0\right)$ motion, while TH-E observed a towards $\left(\mathrm{V}_{\mathrm{N}}<0\right)$ then away $\left(\mathrm{V}_{\mathrm{N}}>0\right)$ motion. This shows that the FTE is not an undulation of the magnetopause but rather a bulge at the magnetopause. By integrating the normal velocity over the period prior to the FTE encounter at probes TH-A and TH-E we determined that the bulge extended $+1700 \mathrm{~km}$ and $-2200 \mathrm{~km}$ from the spacecraft on the magnetosheath or magnetospheric side respectively. Adding that on either side to the TH-A - TH-E spacecraft separation along the magnetopause normal $(\sim 9,900 \mathrm{~km})$ we obtain an FTE thickness of $13,800 \mathrm{~km}$ or about $2.16 \mathrm{R}_{\mathrm{E}}$. This is the first time such observations can be made definitively, and allow us to determine FTE shape, compute total flux content and determine the importance of the presence of the FTE in overall magnetopause energy coupling. Similar, unequivocal observations of an isolated FTE structure are reported by Sibeck et al. (2008). In that case, the observations are made possible due to a fortuitous crossing an isolated FTE by the five THEMIS spacecraft on either side, normal to its motion.

A salient feature of the observations of TH-E is that the spacecraft measured the flow signatures of the approaching FTE and a local magnetospheric pressure enhancement from the time-dependent perturbations of the nearby plasma, even though it never encountered the FTE current or particle populations. This is evidence of remote sensing of an FTE by a satellite on the magnetospheric side, and a counterpart of TH-A's remote sensing of an FTE on the magnetosheath side. These results are possible due to the simultaneous observations of the FTE by TH-E and TH-C closer to the magnetopause and thus able to observe the FTEs that generate these perturbations. The results herein are common in the string-ofpearls THEMIS dataset, and are substantiated using a different event in Liu et al. 2008. 
In summary, analysis of the event on July 12, 2007 using the THEMIS probes from its string-of-pearls configuration shows that (i) FTEs can be remotely sensed using finite gyroradius techniques from the magnetosheath side even in the absence of a crossing of the FTE magnetopause boundary or current layer; (ii) the FTEs can be remotely sensed on the magnetospheric side from observations of the flow and plasma pressure, (iii) the flux rope structure can be revealed using multipoint measurements at separations commensurate to the size of the flux rope, and can be aided by plasma and remote sensing measurements to determine the rope's motion and shape. The scale size of the structures analyzed herein are on the order of $1 \mathrm{R}_{\mathrm{E}}$ but smaller scale sizes can be analyzed with probes TH-C, D and E, which are appropriately positioned in the middle. Although these inner satellites are not optimally separated to study details of the magnetopause current layer, current filaments, or wave particle interactions within FTEs, optimal separations at scales smaller than $1 \mathrm{R}_{\mathrm{E}}$ is the objective of an extended mission.

\section{Summary}

The unique, string-of-pearls configuration during its coast phase enables THEMIS to address questions on substorm and magnetosphere-ionosphere coupling which would not have been addressed otherwise. With a goal to exhibit the power of the THEMIS constellation and the quality of the data, we have presented initial results on two topics in substorm and magnetopause research. The first result, pertaining to the evolution of the substorm expansion phase in space, was obtained on March 23, 2007, when the major substorm intensification occurred at the local time sector of the THEMIS satellites. The probes observed in sequence the substorm dipolarization and Earthward flow bursts, and thus were able to determine that the propagation speed of these current wedge phenomena in space matches the propagation speed of the optical signatures of the westward traveling surge on the ground. Using the finite gyroradius technique of energetic particles we were able to distinguish between the outward expansion of the plasma sheet observed away from the neutral sheet and the westward motion of the dipolarization and Earthward flows. The second result concerns the structure and evolution of FTEs. THEMIS's multiple satellite observations were able to dissect the FTE structure at different distances from the FTE center and ascertain the lateral and radial motion of the FTE, as well as determine its extent and 
differentiate it from a boundary undulation. Using the remote sensing capabilities from the THEMIS SST detector we were able to show that an FTE can be remotely sensed further from the magnetopause, and using the ion data from the ESA instrument we were able to show that an FTE has remote signatures inside the magnetosphere in the plasma pressure and flow velocity even though the FTE boundary is not crossed. Similar analysis techniques employed in the baseline mission started in December of 2007 are expected to enable the resolution of THEMIS’s primary objectives. The power of a string-of-pearls configuration and the capabilities which may arise from a tight but better- controlled clustering of the inner THEMIS probes will both be revisited in a possible extended phase of the mission in the years 2009-2012.

\section{Acknowledgements}

This research was funded by NASA contract NAS5-02099.

\section{References}

Akasofu, S. -I., Physics of magnetospheric substorms, Dordrecht, Netherlands: Reidel, 1976.

Angelopoulos V. et al., Statistical characteristics of bursty bulk flow events, J. Geophys. Res., 99, 21257, 1994.

Angelopoulos, V., et al., Magnetotail flow bursts: association to global magnetospheric circulation, relationship to ionospheric activity and direct evidence for localization, Geophys. Res. Lett., 24, 2271, 1997.

Angelopoulos, V., et al., Multipoint analysis of a bursty bulk flow event on April 11, 1985, J. Geophys. Res., 101, 4967, 1996.

Angelopoulos, V., et al., On the relationship between bursty flows, current disruption and substorms, Geophys. Res. Lett., 26, 2841, 1999.

Angelopoulos, V., The THEMIS Mission, Space Sci. Rev., submitted 2008. (this issue)

Atkinson, G., The current system of geomagnetic bays, J. Geophys. Res., 23, 6063, 1967.

Auster, U. et al., 2008 (this issue).

Baker, D. N., et al., Neutral line model of substorms: Past results and present view, J. Geophys. Res., 101, 12975, 1996.

Bonnell, J., et al., 2008 (this issue).

Carlson, C. W., et al., 2008 (this issue).

Cully, C., et al., 2008 (this issue).

Daly, P. W., et al., Sounding of the plasma sheet in the deep geomagnetic tail using energetic particles, Geoph. Res. Lett., 11, 1070, 1984. 
Fairfield, D. H., et al., Geotail observations of substorm onset in the inner magnetotail, J. Geophys. Res., 103, 103, 1998.

Frank, et al., The Visible Imagin System for the POLAR Spacecraft, Space Sci. Rev., 71, 297, 1995.

Frey, S., et al., 2008 (this issue).

Harris, S., et al., 2008 (this issue).

Harvey, P. R., et al., 2008 (this issue).

Hoffman, R. A., et al., Characteristics of the field-aligned current system in the nighttime sector during auroral substorms, J. Geophys. Res., 99(A11), 21303, 1994.

Horning, B.L., McPherron, R.L., Jackson, D.D., Application of linear inverse theory to a line current model of substorm current systems, J. Geophys. Res., 79 (34): 5202-5210.

Jacquey, C., et al., Location and propagation of the magnetotail current disruption during substorm expansion: analysis and simulation of an ISEE multi-onset event, Geophys. Res. Lett., 3, 389, 1991.

Keiling et al., GRL, submitted, 2008.

Kettmann, G., and P. W. Daly, Detailed determination of the orientation and motion of the plasma sheet boundary layer using energetic protons on ISEE 1 and 2: waves, curves, and flapping, J. Geophys. Res., 93, 7376, 1988.

Larson et al., 2008 (this issue).

Liu et al., GRL submitted, 2008.

Lopez, R. E., and A. T. Y. Lui, A multisatellite case study of the expansion of a substorm current wedge in the near-earth magnetotail, J. Geophys. Res., 95, 8009, 1990.

Lui, A. T. Y., Current disruption in the Earth’s magnetosphere: Observations and models, J. Geophys. Res., 101, 13067, 1996

McFadden, J. P., et al., 2008 (this issue).

McPherron R. et al., Satellite studies of magnetospheric substorms on Aug 15th, 1968, J. Geophys. Res., 78, 3131, 1973.

McPherron, R. L., et al., GRL submitted, 2008.

Mende et al., GRL submitted, 2008.

Mende S., et al., 2008 (this issue).

Nagai, T, Geophys. Monogr. Series, 1991.

Nagai, T., Observed magnetic substorm signatures at synchronous altitudes, J. Geophys. Res., 87, 4405, 1982.

Nakamura, R. et al., Spatial scale of high-speed flows in the plasma sheet observed by Cluster, Geophys. Res. Lett., 31, 9804, 2004.

Ohtani, S. -I., et al., Tail current disruption in the geosynchronous region, in Magnetospheric Substorms, AGU Mongr. Ser., 64, 131, 1991.

Ohtani, S., et al., Radial expansion of the tail current disruption during substorms: A new approach to the substorm onset region, J. Geophys. Res., 97, 3129, 1992.

Ohtani, S.-I., Earthward expansion of tail current disruption: dual-satellite study, J. Geophys. Res., 103, 6815, 1998. 
Press, W. H., et al., Numerical Recipies: The Art of Scientific Computing, Cambridge Univ. Press, 1989.

Rae et al., GRL submitted, 2008.

Roux et al., 2008 (this issue).

Runov et al., GRL, submitted, 2008.

Russell et al., 2008 (this issue).

Russell, C. T., and R. C. Elphic, Initial ISEE magnetometer results: magnetopause observations, Space Sci. Rev., 22, 681, 1978.

Russell, C. T., and R.C. Elphic, ISEE observations of Flux Transfer Events at the dayside magnetopause, Geophys. Res. Lett., 6, 33, 1979.

Sergeev, V. A., et al., Detection of localized, plasma-depleted flux tubes or bubbles in the midtail plasma sheet, J. Geophys. Res., 101, 10817, 1996.

Sergeev, V.A., et al., Comparison of UV optical signatures with the substorm current wedge as predicted by an inversion algorithm, J. Geophys. Res., 101, A2, 2615-2627, 1996.

Shue, J.-H., et al., Magnetopause location under extreme solar wind conditions, J. Geophys. Res., 103, 17691, 1998.

Sibeck D. G., et al., 2008 (this issue)

Strangeway et al., GRL submitted, 2008.

Torr, et al., A Far Ultraviolet Imager for the International Solar Terrestrial Physics Mission, Space Science Reviews, Vol. 71: 329-383, 1995.

Tsyganenko, N. A., A Magnetospheric Magnetic Field Model with a Warped Tail Current Sheet, Planet. Space Sci. 37, 5, 1989.

Tsyganenko, N. A., A model of the near magnetosphere with a dawn-dusk asymmetry: 1. Mathematical structure, J. Geophys. Res., 107(A8), 1179, 2002a

Tsyganenko, N. A., A model of the near magnetosphere with a dawn-dusk asymmetry: 2. Parameterization and fitting to observations, J. Geophys. Res., 107(A8), 1176, 2002b. 